\title{
Comparative analysis and assessment of M. tuberculosis H37Rv protein-protein interaction datasets
}

\author{
Hufeng Zhou', Limsoon Wong ${ }^{2 *}$
}

From Asia Pacific Bioinformatics Network (APBioNet) Tenth International Conference on Bioinformatics - First ISCB Asia Joint Conference 2011 (InCoB/ISCB-Asia 2011)

Kuala Lumpur, Malaysia. 30 November - 2 December 2011

\begin{abstract}
Background: $M$. tuberculosis is a formidable bacterial pathogen. There is thus an increasing demand on understanding the function and relationship of proteins in various strains of $M$. tuberculosis. Protein-protein interactions (PPIs) data are crucial for this kind of knowledge. However, the quality of the main available M. tuberculosis PPI datasets is unclear. This hampers the effectiveness of research works that rely on these PPI datasets. Here, we analyze the two main available $M$. tuberculosis H37Rv PPI datasets. The first dataset is the high-throughput B2H PPI dataset from Wang et al's recent paper in Journal of Proteome Research. The second dataset is from STRING database, version 8.3, comprising entirely of H37Rv PPIs predicted using various methods. We find that these two datasets have a surprisingly low level of agreement. We postulate the following causes for this low level of agreement: (i) the H37Rv B2H PPI dataset is of low quality; (ii) the H37Rv STRING PPI dataset is of low quality; and/or (iii) the H37Rv STRING PPIs are predictions of other forms of functional associations rather than direct physical interactions.
\end{abstract}

Results: To test the quality of these two datasets, we evaluate them based on correlated gene expression profiles, coherent informative GO term annotations, and conservation in other organisms. We observe a significantly greater portion of PPIs in the H37Rv STRING PPI dataset (with score $\geq 770$ ) having correlated gene expression profiles and coherent informative $\mathrm{GO}$ term annotations in both interaction partners than that in the H37Rv B2H PPI dataset. Predicted H37Rv interologs derived from non-M. tuberculosis experimental PPIs are much more similar to the H37Rv STRING functional associations dataset (with score $\geq 770$ ) than the H37Rv B2H PPI dataset. H37Rv predicted physical interologs from IntAct also show extremely low similarity with the H37Rv B2H PPI dataset; and this similarity level is much lower than that between the S. aureus MRSA252 predicted physical interologs from IntAct and S. aureus MRSA252 pull-down PPIs. Comparative analysis with several representative two-hybrid PPI datasets in other species further confirms that the H37Rv B2H PPI dataset is of low quality. Next, to test the possibility that the H37Rv STRING PPIs are not purely direct physical interactions, we compare M. tuberculosis H37Rv protein pairs that catalyze adjacent steps in enzymatic reactions to B2H PPIs and predicted PPIs in STRING, which shows it has much lower similarities with the B2H PPIs than with STRING PPIs. This result strongly suggests that the H37Rv STRING PPIs more likely correspond to indirect relationships between protein pairs than to B2H PPIs. For more precise support, we turn to S. cerevisiae for its comprehensively studied interactome. We compare S. cerevisiae predicted PPIs in STRING to three independent protein relationship datasets which respectively comprise PPIs reported in $\mathrm{Y} 2 \mathrm{H}$ assays, protein pairs reported to be in the same protein complexes, and protein pairs that catalyze successive reaction steps in enzymatic reactions. Our analysis reveals that $S$. cerevisiae predicted STRING PPIs have much

\footnotetext{
*Correspondence: wongls@comp.nus.edu.sg

${ }^{2}$ School of Computing, National University of Singapore, 13 Computing

Drive, Singapore 117417

Full list of author information is available at the end of the article
}

\section{Ciomed Central}

(c) 2011 Zhou and Wong; licensee BioMed Central Ltd. This is an open access article distributed under the terms of the Creative Commons Attribution License (http://creativecommons.org/licenses/by/2.0), which permits unrestricted use, distribution, and reproduction in any medium, provided the original work is properly cited. 
higher similarity to the latter two types of protein pairs than to two-hybrid PPIs. As H37Rv STRING PPIs are predicted using similar methods as S. cerevisiae predicted STRING PPIs, this suggests that these H37Rv STRING PPIs are more likely to correspond to the latter two types of protein pairs rather than to two-hybrid PPIs as well.

Conclusions: The H37Rv B2H PPI dataset has low quality. It should not be used as the gold standard to assess the quality of other (possibly predicted) H37Rv PPI datasets. The H37Rv STRING PPI dataset also has low quality; nevertheless, a subset consisting of STRING PPIs with score $\geq 770$ has satisfactory quality. However, these STRING "PPIs" should be interpreted as functional associations, which include a substantial portion of indirect protein interactions, rather than direct physical interactions. These two factors cause the strikingly low similarity between these two main H37Rv PPI datasets. The results and conclusions from this comparative analysis provide valuable guidance in using these M. tuberculosis H37Rv PPI datasets in subsequent studies for a wide range of purposes.

\section{Background}

Each year millions of tuberculosis patients perish, and fully one-third of the world's population is infected with the causative agent of this disease, $M$. tuberculosis[1]. $M$. tuberculosis $\mathrm{H} 37 \mathrm{Rv}$ is one of first fully sequenced $M$. tuberculosis strains [2]. With the increasingly intensive research focused on this pathogen around the world, $M$. tuberculosis $\mathrm{H} 37 \mathrm{Rv}$ protein-protein interaction (PPI) data has become an important source of protein function and relationship information for relevant studies in microbiology, molecular biology, computational biology and medicine. However, M. tuberculosis H37Rv PPI data is far from complete and accurate. Hitherto predicted M. tuberculosis H37Rv PPIs in the STRING database (version 8.3 contains 248,574 PPIs covering 3,965 proteins in $\mathrm{H} 37 \mathrm{Rv}$ ) [3] have seen the most frequent used [4-6] because large-scale experimental PPI datasets have not been available until recently. The first large-scale proteome-wide PPI dataset of $\mathrm{H} 37 \mathrm{Rv}$ was produced in 2010 using a high-throughput bacterial two-hybrid (B2H) approach [7]; it comprises 8,042 PPIs covering 2,907 proteins. No doubt in the foreseeable future, increasingly more studies on $M$. tuberculosis will be based on both of these datasets.

There is an extremely low overlap of just 276 proteinprotein interactions shared between the $8,042 \mathrm{H} 37 \mathrm{Rv}$ PPIs in the B2H dataset and the 248,574 predicted H37Rv PPIs in STRING. It is the objective of this work to investigate the cause of this unexpectedly low overlap. We hypothesize that this low overlap between the two datasets may be due to (i) the $\mathrm{B} 2 \mathrm{H}$ dataset is poor in quality, (ii) the STRING dataset is poor in quality, and/ or (iii) the STRING dataset does not correspond to direct physical protein-protein interactions.

In order to test the quality of these two M. tuberculosis H37Rv PPI datasets, we evaluate them based on correlated gene expression profiles, coherent informative GO term annotations, and conservation in other organisms. Two proteins that interact are expected to be expressed at the same time and space; thus their underlying genes are likely to exhibit correlated expression profiles. Two proteins interact to effect a biological process or molecular function; thus they are expected to be annotated to some GO terms in common or GO terms that are closely related. Many protein-protein interactions are expected to be conserved across several organisms that have common ancestry; thus real protein interactions are likely to coincide with interologs from such organisms.

These assessments indicate that H37Rv B2H PPIs agree less well with correlated gene expression profiles, coherent informative GO term annotations, and conservation in other organisms than H37Rv STRING PPIs (with score $\geq 770$ ). This suggests that PPIs in the $\mathrm{H} 37 \mathrm{Rv} \mathrm{B} 2 \mathrm{H}$ dataset may contain a high level of noise (false positives).

S. aureus is a bacterial pathogen with available highthroughput experimental physical PPI data; and it is close to M. tuberculosis. Thus interologs conserved in $S$. aureus should be very likely conserved in $M$. tuberculosis. However, H37Rv predicted physical interologs from IntAct show quite low similarity with the H37Rv B2H PPI dataset; and this similarity level is lower than that between the $S$. aureus MRSA252 predicted physical interologs from IntAct and $S$. aureus MRSA252 pulldown PPIs. This suggests the $\mathrm{H} 37 \mathrm{Rv}$ B2H dataset may also be missing many real PPIs (false negatives).

To further confirm the suspected low quality of the H37Rv B2H PPI dataset, we compare this M. tuberculosis $\mathrm{H} 37 \mathrm{Rv} \mathrm{B} 2 \mathrm{H}$ dataset with representative two-hybrid PPI datasets in other species (C. jejuni, Synechocystis and $S$. cerevisiae) [8-10]. These comparative analyses demonstrate that the quality of the M. tuberculosis H37Rv B2H PPI dataset is poorer than other two-hybrid PPI datasets.

As mentioned earlier, the H37Rv STRING PPI dataset (with score $\geq 770$ ), comprising entirely of PPIs predicted using a variety of methods, shows good agreement with correlated gene expression profiles, coherent informative GO term annotations, and conservation in other organisms. However, protein pairs that are functionally linked are also expected to agree well with 
correlated gene expression profiles, coherent informative GO term annotations, and conservation in other organisms, even though many functionally-linked protein pairs do not have direct physical interactions. In order to test whether the predicted PPIs in STRING correspond to direct physical protein-protein interactions, we should analyze the similarity between these predicted PPIs with several distinct types of protein pairs such as experimental PPIs obtained from two-hybrid assays, protein pairs that belong to the same protein complexes, and protein pairs that catalyze adjacent steps in enzymatic pathways. As these types of additional information are not available for $M$. tuberculosis, we turn to the model organism $S$. cerevisiae where more comprehensive information is available. We extract from STRING an unbiased representative $S$. cerevisiae PPI subset (which we denote "predicted functional associations dataset") that are predicted using similar methods as the H37Rv STRING PPI dataset. For the three different types of protein pairs, we use the following gold standard: (i) the S. cerevisiae two-hybrid PPI dataset from $\mathrm{Yu}$ et al [10], (ii) all protein pairs found in the same $S$. cerevisiae protein complexes from Wodak Lab [11], and (iii) protein/gene pairs that catalyze/form successive reaction steps in biological pathways from KEGG [12], WikiPathways [13,14] and BioCyc [15].

This analysis indicates that the predicted $S$. cerevisiae STRING PPIs show higher similarities with protein pairs in the same protein complexes and protein/gene pairs that catalyze/form adjacent reaction steps in biological pathways than with PPIs reported in two-hybrid assays. Therefore, the predicted S. cerevisiae STRING PPIs are mostly not direct physical protein-protein interactions. As the H37Rv STRING PPIs are predicted using similar methods, in turn, they are also unlikely to correspond to direct physical interactions. Nonetheless, their relatively good agreement with correlated gene expression profiles, coherent informative GO term annotations, and conservation in other organisms suggest that the H37Rv STRING PPIs (at score $\geq 770$ ) are proteins that are functionally linked.
This work thus provides an important guidance to the researchers who might base their works on the two $M$. tuberculosis H37Rv PPI datasets. The details of our analyses are presented in the sections below.

\section{Results}

This section can be divided into four parts: (i) Discover the low similarity between the two main H37Rv PPI datasets. (ii) Evaluate the quality of the two H37Rv PPI datasets in the same organism. (iii) Assess the quality of the H37Rv B2H PPI dataset across organisms. (iv) Analyse characteristics of the STRING PPIs in M. tuberculosis and S. cerevisiae.

\section{Lack of agreement between the two M. tuberculosis H37Rv PPI datasets}

The H37Rv B2H PPI dataset is used as benchmark and different subsets of H37Rv STRING PPIs are tested against it. We consider all H37Rv STRING PPIs as well as STRING H37Rv PPIs based on specific methods (gene neighbourhood, gene fusion, etc.; details are listed in Table 1) used for predicting them. For each subset, in Figure 1, we show the Jaccard coefficient, precision, and recall of each predicted subset at STRING score $\geq 770$. According to Figure 2, STRING score threshold at around 770 generally maximizes the overlap between two-hybrid PPIs and STRING predicted PPIs in $M$. tuberculosis H37Rv. It is clear from Figure 1 that the STRING PPIs predicted by various methods all have extremely low precision, recall, and overlap with the $\mathrm{H} 37 \mathrm{Rv}$ B2H PPI dataset. Below are some representative statistics:

Overlapping PPIs between the two datasets: 276.

STRING PPIs Precision: 0.00215 Recall: 0.03503.

STRING PPIs (at score $\geq 770$ ) Precision: 0.00574 Recall: 0.00896.

The extremely low agreement between the H37Rv PPIs in the STRING and B2H PPI datasets is rather unexpected. We hypothesize that it may be a result of one or more of the following situations. First, it may be that the H37Rv B2H PPI dataset contains an unusually

Table 1 Summary of number of PPIs in each STRING prediction approaches

\begin{tabular}{llll}
\hline STRING database prediction method & Number of PPIs & STRING database prediction method \\
\hline Neighbourhood & 12,706 & Transferred neighborhood \\
Co-expression & 0 & Transferred co-expression & Number of PPIs \\
Experiments & 4 & Transferred experimental & T8,376 \\
Databases & 7,030 & Transferred databases & Transferred text mining \\
Text mining & 2,715 & Co-occurrence & \\
Gene fusion & 2,646 & & \\
\hline All STRING database PPIs & 248,574 & &
\end{tabular}

Summary of various subsets of H37Rv PPIs in STRING and their sources. 


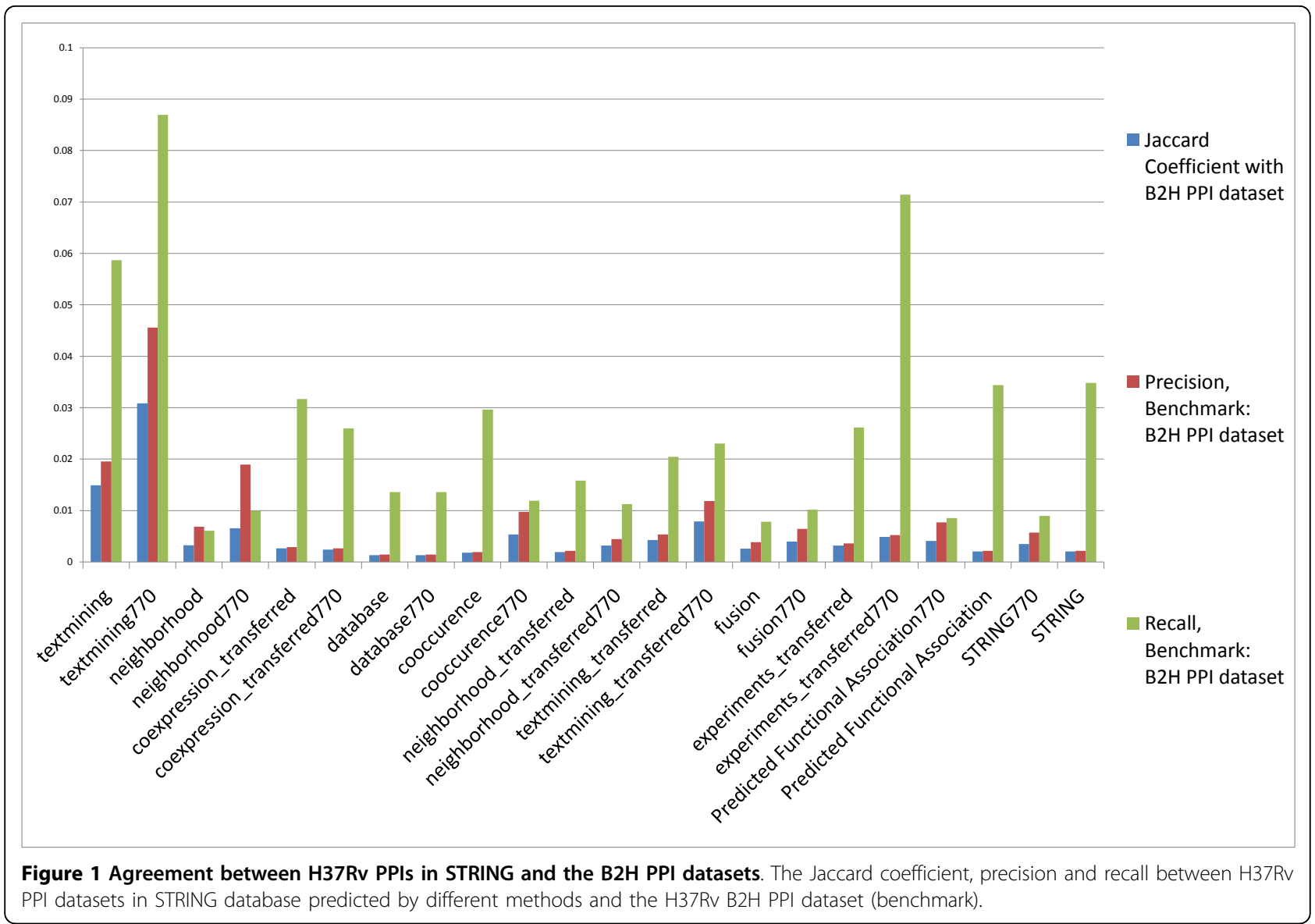

high level of noise. Second, it may be that the H37Rv STRING PPI dataset and subsets thereof contain an unusually high level of noise. Third, it may be that the predicted PPIs in STRING are not direct physical interactions; rather, they may primarily be other types of functional associations such as protein pairs in the same protein complexes and enzyme pairs catalyzing successive reaction steps.

\section{Overlap PPI number ratios at various STRING score thresholds}

The results above reveal the surprisingly low coverage between the two H37Rv PPI datasets. However, as shown in Figure 2, at STRING score $\geq 770$, there is a higher level of overlap between the two datasets. This increase in overlap between two-hybrid PPI dataset and STRING predicted functional associations dataset at high scores is also observed in C. jejuni[8], Synechocystis [9] and S. cerevisiae[10]. This suggests that STRING PPIs with high score potentially has higher quality than STRING PPIs with a lower score. Nevertheless, the overlap between these two-hybrid PPI datasets and their respective STRING predicted functional association datasets is no more than $8 \%$ at any score interval. Thus, even at a high STRING score threshold, there is no clear agreement between two-hybrid PPIs and STRING predicted functional association datasets. Assuming that not all of these two-hybrid PPI datasets are of low quality, this lack of clear agreement strongly suggests that STRING predicted PPIs are unlikely to correspond mainly to direct physical interactions.

\section{Analysis of PPI dataset using PPI functional intensity matrix}

We next use PPI functional intensity matrix to visualize the functional relationship between the two interacting proteins in PPIs. Figure 3 shows that the H37Rv STRING PPI dataset (with score $\geq 770$ ) has strong intensity at the diagonal of its PPI functional intensity matrix. This means a substantial portion of PPIs in this dataset have both partners in the same function category. In contrast, both the H37Rv B2H PPI dataset and the H37Rv STRING PPI dataset (without thresholding at score $\geq 770$ ) exhibit weak intensity at the diagonal of its matrix. Moreover, both the H37Rv B2H PPI dataset and the H37Rv STRING PPI dataset (without thresholding at score $\geq 770$ ) have substantial amounts of PPIs involving proteins that are functionally unclear or uninformative. 

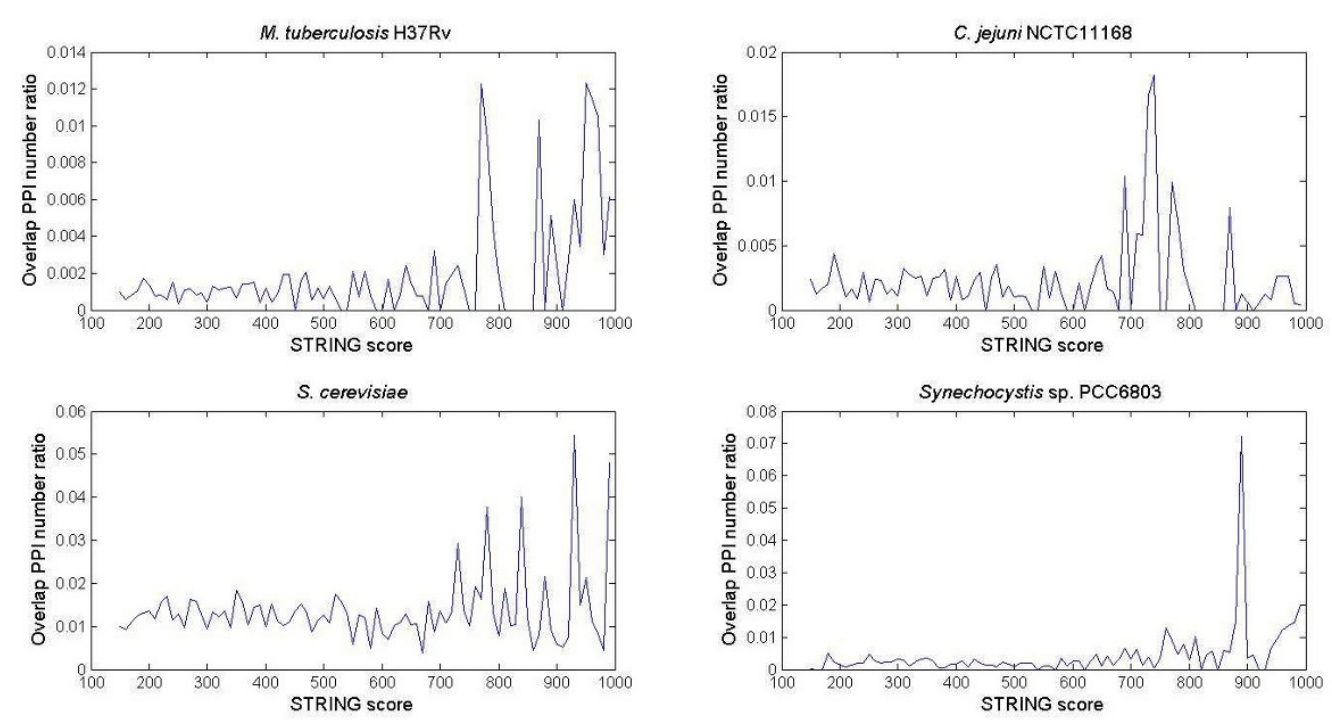

Figure 2 Overlap PPI number ratios at various STRING score thresholds The overlap PPI number ratios at various STRING score thresholds between (i) the H37Rv B2H PPI dataset and the H37Rv STRING predicted functional associations dataset, (ii) the S. cerevisiae Y2H PPI dataset and the S. cerevisiae STRING predicted functional associations dataset, (iii) the C. jejuni NCTC11168 Y2H PPI dataset and the C. jejuni NCTC11168 STRING predicted functional associations dataset, and (iv) the Synechocystis sp. PCC6803 Y2H PPI dataset and Synechocystis sp. PCC6803 STRING predicted functional associations dataset.

\section{Assessment of PPI datasets using informative GO terms}

The functional intensity matrix visualisation in Figure 3 only provides broad perspectives on the functional distribution of PPI datasets. It is not sufficient for gauging the quality of the datasets. Two interacting proteins are more likely to be localized in the same cellular component and/or having a common function role than not [16]. So we calculate the percentage of PPIs in a PPI dataset having coherent informative GO terms-i.e., the rate of interacting protein pairs with common function roles (measured based on informative GO terms in MF and BP categories) and cellular localization (measured based on informative $\mathrm{GO}$ terms in the $\mathrm{CC}$ category) in the PPI dataset-to evaluate the quality of the PPI dataset.

The results of $M$. tuberculosis $\mathrm{H} 37 \mathrm{Rv}$ GO term annotation and informative GO term identification are summarized in Table 2. The percentage of PPIs having coherent informative GO terms is computed for each of the datasets in Figure 4. The datasets include subsets of STRING derived from specific source channels in STRING. Note that some source channels may introduce GO-related information into STRING. In particular, the "database" and "database transfer" channels may

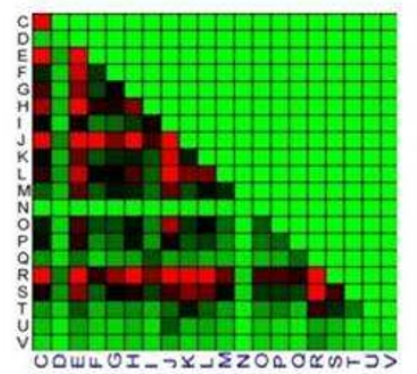

(i) H37Rv STRING PPIS

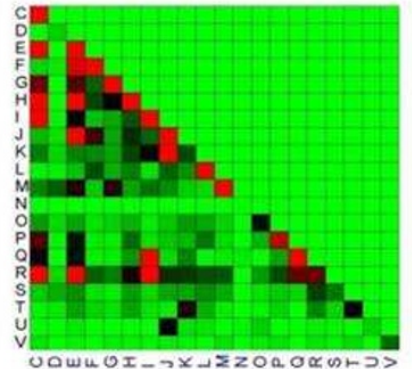

(ii) H37Rv STRING PPIs at score $\geq 770$

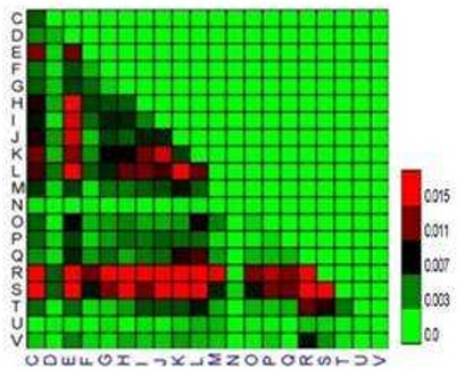

(iii) H37Rv B2H PPIS

Figure 3 PPIs functional intensity matrix analysis of the PPI datasets. Functional intensity matrices of (i) H37Rv STRING PPI dataset, (ii) H37Rv STRING PPI dataset at score $\geq 770$, and (iii) H37Rv B2H PPI dataset. The "C", "D", "E", etc. in the axes of these matrices are COG functional categories. 
Table 2 M. tuberculosis H37Rv GO term annotation and informative GO term identification

\begin{tabular}{lccc}
\hline & No. of informative GO term & No. of proteins been annotated with GO term & No. of proteins been annotated with informative GO term (N = 30) \\
\hline CC & 3 & 563 & 233 \\
BP & 32 & 1822 & 1200 \\
MF & 32 & 2034 & 1198 \\
GO & 67 & 2225 & 1659 \\
\hline
\end{tabular}

This table summarizes the result of $M$. tuberculosis $\mathrm{H} 37 \mathrm{Rv} \mathrm{GO}$ term annotation and informative GO term identification.

collect PPIs derived from Protein Complexes in the Gene Ontology (GO) database. Thus, to avoid circularity in our results here and elsewhere, we mainly use statistics from an unbiased subset "predicted functional associations dataset" of STRING obtained by excluding the PPIs from source channels that may introduce confounding factors. The "predicted functional associations dataset" consists of STRING PPIs that are generated only from the following prediction approaches: gene neighborhood, transferred neighborhood, gene fusion, and co-occurrence, transferred co-expression, text mining, and transferred text mining. Among the three categories of GO terms, the datasets generally show a high percentage of coherence with respect to informative CC GO terms. However, this observation should be dismissed because there are only three distinct informative CC GO terms, which is an order of magnitude less than informative MF and BP GO terms; see Table 2. The random PPI dataset has the lowest percentage of PPIs with coherent informative GO terms in all the tested PPI datasets, which makes sense. But the H37Rv
B2H PPI dataset has the second lowest percentage of PPIs with coherent informative GO terms and is very close to the random PPI dataset. This indicates that the M. tuberculosis H37Rv B2H PPI dataset has the lowest quality among all the PPI datasets been evaluated. The $\mathrm{H} 37 \mathrm{Rv}$ predicted functional association dataset (without thresholding at score $\geq 770$ ) also has a low percentage of PPIs with coherent informative GO terms and is thus of low quality. However, most PPI datasets from STRING (with score $\geq 770$ ) show a much higher percentage of PPIs having coherent informative GO terms than the H37Rv B2H PPI dataset, suggesting that a higher percentage of PPIs in these datasets may have better reliability than those of the B2H PPI dataset and of the STRING PPI dataset as a whole.

\section{Analysis of PPI datasets using gene expression profile correlation}

Two proteins that interact are more likely to be correlated in the expression of their underlying genes than not [17]. In fact, co-expression is one of the prediction

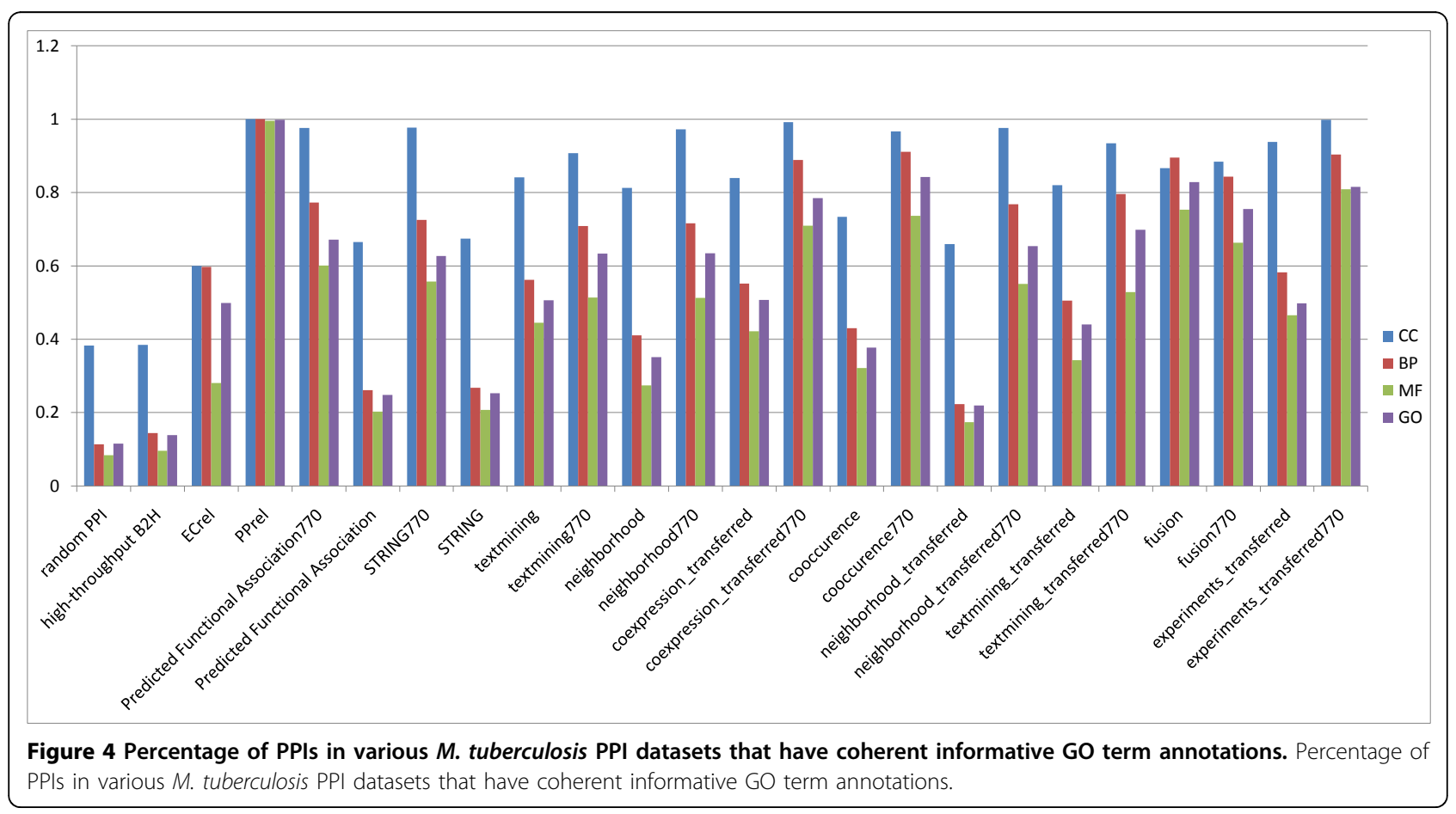


methods in STRING [3]. However, Table 1 shows no STRING PPI predicted from co-expression in $M$. tuberculosis H37Rv. Given that M. tuberculosis H37Rv gene expression data is readily available in public repositories (e.g., NCBI Gene Expression Omnibus [18]), this lack of H37Rv PPIs predicted using this information is an unexpected limitation of STRING. At the same time, this absence makes using correlation of gene expression profiles for assessing the quality of the $\mathrm{H} 37 \mathrm{Rv} \mathrm{B} 2 \mathrm{H}$ and STRING PPI datasets unbiased. The results in Figure 5 clearly show that the H37Rv STRING PPI dataset (at score $\geq 770$ ) has a much larger proportion of PPIs that exhibit correlation in the expression profiles of their underlying genes than the H37Rv B2H PPI dataset and the whole H37Rv STRING PPI dataset. In fact, a mere 223 PPIs in the H37Rv B2H PPI dataset have significant correlated gene expression profiles (Pearson's correlation coefficient $>0.4$ ). These 223 PPIs are likely to be more reliable than most of the other PPIs in the $\mathrm{H} 37 \mathrm{Rv} \mathrm{B} 2 \mathrm{H}$ dataset.

\section{PPI datasets assessment using predicted interologs from STRING experimental PPIs}

As PPIs are often conserved by related organisms, homology transfer is an often-used technique to predict PPIs-the so-called interologs. On the one hand, a higher level of agreement that a PPI dataset has with interologs gives us confidence that the PPIs are more reliable-i.e., fewer false positives-as they are conserved in other organisms. On the other hand, a lower level of agreement signals that PPIs conserved in related organisms are potentially missing-i.e., more false negativesin the PPI dataset.

We use different homology transfer methods to build a set of predicted interologs in the target organism $M$. tuberculosis $\mathrm{H} 37 \mathrm{Rv}$ from other (non-M. tuberculosis) organisms that have experimental PPIs in STRING. We then compare this set of interologs to both the H37Rv STRING predicted functional associations dataset (with score $\geq 770$ ) and H37Rv B2H PPI dataset. We use this subset of STRING PPIs (predicted functional associations dataset) to avoid possible confounding effects because the full set of H37Rv STRING PPIs contains some PPIs that actually originate from interologs predicted from experimental PPIs.

In order to find better source PPIs for predicting interologs, a more reliable subset of the experimental PPIs in STRING has been chosen in this experiment. This subset consists of the experimental PPIs in STRING that also have STRING prediction supports.

Table 3 shows the results of interologs predicted from STRING experimental PPIs, while Table 4 shows the results of interologs predicted from the reliable subset of STRING experimental PPIs. Under the same method and benchmark, Jaccard coefficient, precision and recall in Table 4 are much higher than that in Table 3. This demonstrates STRING experimental PPIs with prediction supports is a better source than the whole STRING experimental PPIs for predicting interologs. Because experimental PPIs also have significant noise in most
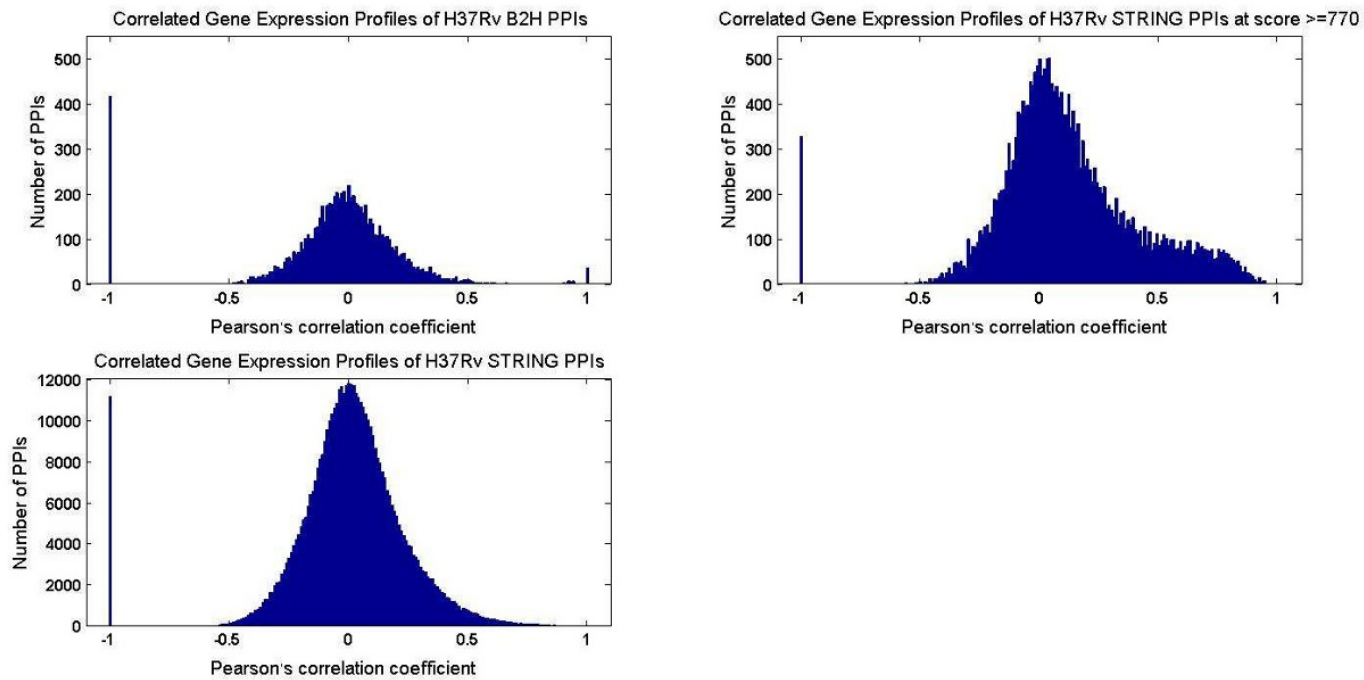

Figure 5 PPI datasets assessment by gene expression profile correlation. The distribution of Pearson's correlation coefficient of the expression profiles of underlying genes of different PPI datasets are given in this figure ( $x$ axis is the Pearson's correlation coefficient, $y$ axis is the number of PPIs). The bar at -1 in the charts here corresponds to PPIs where we do not have the expression profiles of their underlying genes. 
Table 3 Results of predicted interologs from STRING experimental PPIs

\begin{tabular}{|c|c|c|c|}
\hline \multirow{2}{*}{$\frac{\text { Source PPIs for homology transfer }}{\text { Identify homology and transfer }}$} & \multicolumn{3}{|c|}{ STRING database experimental PPIs } \\
\hline & PIDE $>30$ & HVAL $>20$ & Orthologues \\
\hline & Coverage $>0.2$ & E-VALUE $<1^{*} \mathrm{e}-6$ & Identified by Inparanoid (default parameters) \\
\hline & E-VALUE $<1 * e-10$ & & \\
\hline Benchmark & \multicolumn{3}{|c|}{ M.tuberculosis H37Rv high-throughput B2H PPIs dataset } \\
\hline Jaccard coefficient & 0.00354 & 0.00187 & 0.00289 \\
\hline Precision & 0.00375 & 0.00196 & 0.00339 \\
\hline Recall & 0.0588 & 0.0384 & 0.0190 \\
\hline Overlapping PPIs & 5 & 4 & 5 \\
\hline Benchmark & \multicolumn{3}{|c|}{ M.tuberculosis H37Rv predicted functional associations dataset from STRING database(with PPIs score above 770) } \\
\hline Jaccard coefficient & 0.285 & 0.179 & 0.129 \\
\hline Precision & 0.355 & 0.236 & 0.239 \\
\hline Recall & 0.625 & 0.422 & 0.219 \\
\hline Overlapping PPIs & 656 & 830 & 525 \\
\hline
\end{tabular}

Predicted interologs from STRING experimental PPIs and a summary of Jaccard coefficient, precision and recall between the interologs datasets and two benchmarks.

organisms, choosing the subset with both experiments and STRING prediction supports would have better reliability. When switching benchmark from the H37Rv $\mathrm{B} 2 \mathrm{H}$ PPI dataset to the H37Rv STRING predicted functional associations dataset (with score $\geq 770$ ), the resulting Jaccard coefficient, precision, recall, and overlapping PPIs number, increase significantly (about two orders of magnitude). This clearly demonstrates that the predicted $M$. tuberculosis $\mathrm{H} 37 \mathrm{Rv}$ interologs from other (non-M. tuberculosis) organisms' experimental PPIs in STRING are in much lower agreement with the H37Rv B2H PPI dataset than with the H37Rv STRING predicted functional associations dataset (with score $\geq 770$ ). In particular, depending on the method used for constructing interologs, just 4-5 interologs are found in the $\mathrm{H} 37 \mathrm{Rv}$ B2H PPI dataset, while 524-830 interologs are found in the H37Rv STRING predicted functional associations dataset (with score $\geq 770$ ); see Table 4 . This suggests that the H37Rv B2H PPI dataset may have a high number of false negatives.

PPI datasets assessment using predicted interologs from IntAct prokaryotic physical interactions

The results discussed above are already persuasive but not precise enough to make sufficient judgment. In particular, the experimental PPIs in STRING are a mixture of both experimental physical interactions and experimental functional associations (e.g., genetic interactions). This may introduce some bias towards the larger agreement observed with the STRING predicted functional associations dataset. Furthermore, some source experimental PPIs in the dataset are not from organisms close

Table 4 Results of predicted interologs from STRING experimental PPIs (with other STRING database prediction support)

\begin{tabular}{cccc}
\hline Source PPIs for homology transfer & \multicolumn{2}{c}{ STRING database experimental PPIs (with other prediction support) } \\
\hline Identify homology and transfer & $\begin{array}{c}\text { PIDE }>30 \\
\text { Coverage }>0.2 \\
\text { E-value }<1^{*} \text { e- } 10\end{array}$ & $\begin{array}{c}\text { HVAL }>20 \\
\text { E-value }<1^{*} \text { e- } 6\end{array}$ & $\begin{array}{c}\text { Orthologues } \\
\text { Identified by inparanoid (default parameters) }\end{array}$ \\
\hline Benchmark & & M.tuberculosis H37Rv high-throughput B2H PPIs dataset \\
\hline Jaccard coefficient & 0.00485 & 0.00225 & 0.00359 \\
Precision & 0.00507 & 0.00267 & 0.00401 \\
Recall & 0.100 & 0.0563 & 0.0331 \\
Overlapping PPIs & 5 & 4 & 4 \\
\hline Benchmark & M.tuberculosis H37Rv predicted functional associations datasetfrom STRING database (with PPIs score above 770) \\
\hline Jaccard coefficient & 0.365 & 0.233 & 0.186 \\
Precision & 0.467 & 0.314 & 0.365 \\
Recall & 0.625 & 0.473 & 0.275 \\
Overlapping PPIs & 651 & 828 & 524
\end{tabular}

Predicted interologs from STRING experimental PPIs (with other STRING database prediction support) and a summary of Jaccard coefficient, precision and recall between the interologs datasets and two benchmarks. 
to M. tuberculosis. To control for these potential confounding factors, we conduct another set of experiments using interologs predicted from the following two sources that-in theory-are closer to the characteristics of H37Rv B2H PPIs. The first source is the latest pulldown PPI dataset in S. aureus MRSA252 [19]. This dataset has just been released and is not yet included in the current STRING database (version 8.3). The second source comprises physical interactions of eight bacteria (mostly pathogens) in the IntAct database [20]. In particular, we have chosen Rickettsia sibirica 246, Escherichia coli K12, Campylobacter jejuni, Treponema pallidum, Synechocystis sp. PCC 6803, Mycoplasma pneumonia, Myxococcus xanthus DK 1622, and Streptococcus pneumoniae. We group the physical interactions of these eight bacteria from IntAct [20] into the "selected IntAct prokaryotic physical interactions dataset".

We transfer interologs from the "selected IntAct prokaryotic physical interactions dataset" to $M$. tuberculosis H37Rv (named "H37Rv physical interologs dataset") and to S. aureus MRSA252 (named "MRSA252 physical interologs dataset"). We also transfer interologs from the $S$. aureus MRSA252 high-throughput pull-down PPI dataset to M. tuberculosis H37Rv (named "H37Rv pulldown interologs dataset"). All the homolog identifications of this experiment use the condition PIDE $>30$, Coverage $>0.2$, E-VALUE $<1 * \mathrm{e}-10$.

The comparative analyses results are given in Table 5 . The results clearly demonstrate that the H37Rv physical interologs dataset is much more similar to the H37Rv pull-down interologs dataset than to the $\mathrm{H} 37 \mathrm{Rv} \mathrm{B} 2 \mathrm{H}$ PPI dataset. Moreover, the similarity between $S$. aureus MRSA252 physical interologs dataset and pull-down PPI dataset is also much higher than that between H37Rv physical interologs dataset and H37Rv B2H PPI dataset. These results further strengthen the conclusion that the H37Rv B2H PPI dataset has poor quality.

\section{Quality evaluation of two-hybrid PPI datasets from several organisms}

In the discussions above, we have concluded that the quality of the M. tuberculosis H37Rv B2H PPI dataset is low in quality. However, it is generally believed that two-hybrid PPI datasets do not have high reliability because of their high false positives and false negative rates. It is thus of interest to determine whether this H37Rv B2H PPI dataset is even worse than other twohybrid PPI datasets in terms of quality. So we compare the M. tuberculosis H37Rv B2H PPI dataset to several representative two-hybrid PPI datasets in other organisms-viz., C. jejuni, Synechocystis and S. cerevisiae [8-10]-by calculating their percentage of PPIs having coherent informative GO terms. The results are shown in Figure 6. As these organisms have a large difference in the number of informative GO terms and in the distribution of GO term annotations to their proteins, we also calculate the ratio of the percentage of coherently annotated PPIs in the dataset of an organism to that of appropriately generated random PPI datasets. The results are shown in Table 6 . In particular, the ratios for $M$. tuberculosis, C. jejuni, Synechocystis and S. cerevisiae are respectively 1.20 , $1.28,3.03$, and 5.73. These results sufficiently demonstrate that, compared to other representative twohybrid PPI datasets, the M. tuberculosis H37Rv B2H PPI dataset has distinctly lower percentage of coherently annotated PPIs.

We have also computed for each two-hybrid PPI dataset its similarity to the corresponding predicted functional associations dataset from STRING; see Figure 7. These results indicate the H37Rv B2H PPI dataset also has the lowest similarity to the corresponding STRING predicted functional association dataset.

\section{Analysis of the characteristics of M. tuberculosis H37Rv PPIs using integrated pathway gene pair relationships} From the results presented earlier, it seems that many H37Rv STRING PPIs may not be direct physical interactions. In order to understand what these PPIs may better correspond to, we collect pair-wise protein/gene relationships (mainly the ECrel and PPrel datasets, see Table 7) from several major pathway databases, and compare them with the various PPI datasets considered earlier in this paper. The ECrel dataset comprises enzyme pairs that catalyze successive reaction steps in enzymatic pathways. The PPrel dataset comprises more direct protein-protein interactions but it also contains protein pairs in the same complexes. Thus, a PPI dataset

Table 5 Comparison of physical interactions and predicted physical interologs datasets

\begin{tabular}{lllllll}
\hline Organism & Benchmark dataset & Testing PPI dataset & Jaccard coefficient & Precision & Recall & Overlapping PPIs No. \\
\hline M. tuberculosis H37Rv & H37Rv pull-down interologs & H37Rv physical interologs & 0.021 & 0.205 & 0.023 & 40 \\
M. tuberculosis H37Rv & H37Rv B2H PPI & H37Rv physical interologs & 0.005 & 0.005 & 0.050 & 2 \\
S. aureus MRSA252 & MRSA252 Pull Down PPI dataset & MRSA252 physical interologs & 0.019 & 0.126 & 0.022 & 23 \\
\hline
\end{tabular}

Summary of the comparison results between physical interactions and predicted interologs datasets. "H37Rv physical interologs dataset" consists of interologs transferred from "selected IntAct prokaryotic physical interactions dataset" to M. tuberculosis H37Rv; "MRSA252 physical interologs dataset" consists of interologs transferred from "selected IntAct prokaryotic physical interactions dataset" to S. aureus MRSA252; "H37Rv pull-down interologs dataset" consists of interologs transferred from "S. aureus MRSA252 high-throughput pull-down PPI dataset" to M. tuberculosis H37Rv. 


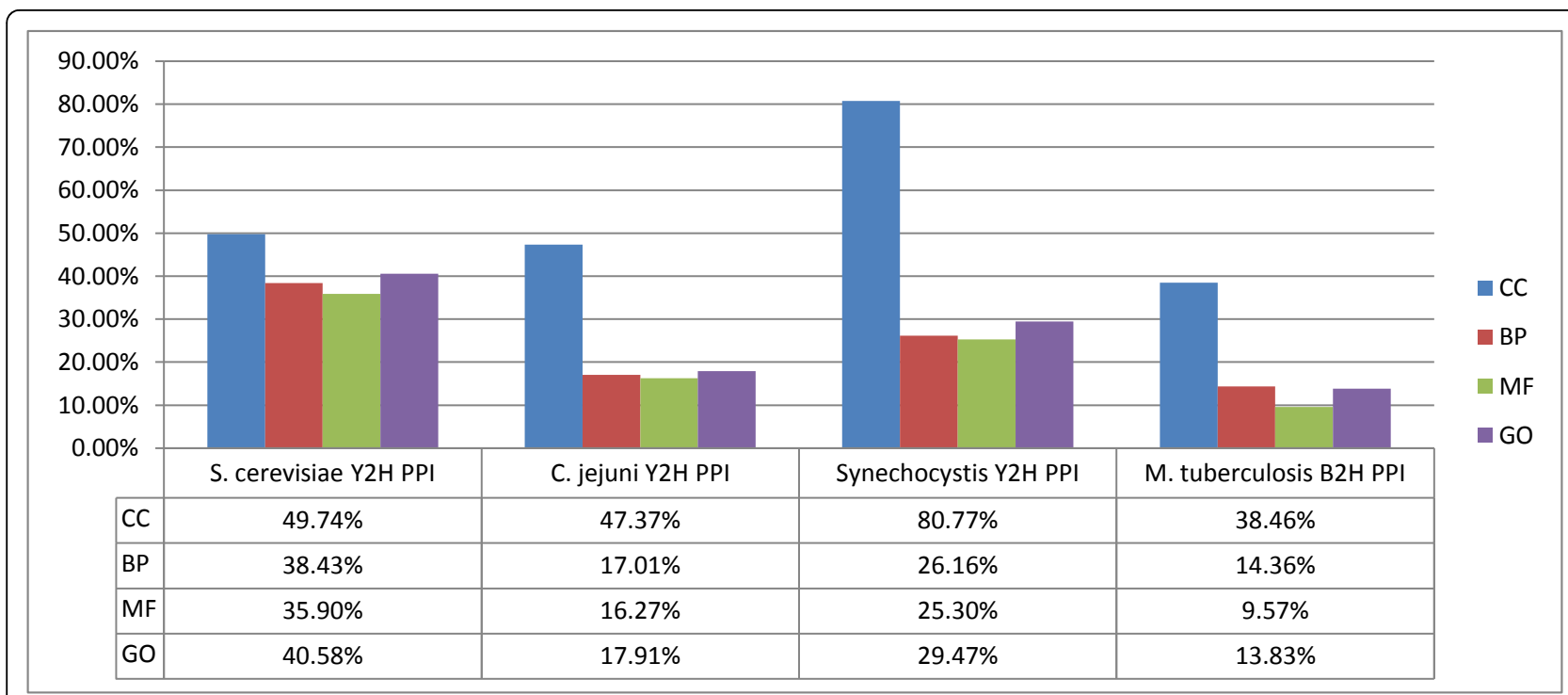

Figure 6 Percentage of PPIs in representative two-hybrid PPI datasets having coherent informative GO terms. The histogram is a summary of the percentage of PPIs having coherent informative GO terms in each of the representative two-hybrid PPI datasets in four organisms.

containing more indirect protein relationships should show high similarity to the ECrel dataset.

However, this task is hampered by the sparse information stored in all the current main pathway databases, like KEGG [12], WikiPathways [13,14] and BioCyc [15]. Therefore an integration of pathway information from the three main databases is needed to maximize the effectiveness of pathway information for this comparative analysis of PPI datasets.
The number of pathways obtained from the three major pathway databases [12-15], and the number of pathways after integration are listed in Table 8 . All together, the M. tuberculosis $\mathrm{H} 37 \mathrm{Rv}$ integrated pathway gene pair relationships contain 230 pathways, comprising 3,573 gene pairs involving 668 proteins.

In the M. tuberculosis $\mathrm{H} 37 \mathrm{Rv}$ integrated pathway gene pair relationships, most of the gene pair relationships are ECrel (2,527 pairs) and PPrel (916 pairs), other type of

Table 6 Percentage of PPIs in representative two-hybrid PPI datasets having coherent informative GO terms

\begin{tabular}{|c|c|c|c|c|}
\hline & CC & $\mathrm{BP}$ & MF & $\mathrm{GO}$ \\
\hline S. cerevisiae Y2H PPI & $49.74 \%$ & $38.43 \%$ & $35.90 \%$ & $40.58 \%$ \\
\hline S. cerevisiae random PPI & $5.03 \%$ & $3.70 \%$ & $7.72 \%$ & $7.08 \%$ \\
\hline Info $\mathrm{GO}$ ratio of $\mathrm{S}$. cerevisiae (Y2H PPI/ random) & 9.88 & 10.38 & 4.65 & 5.73 \\
\hline Info GO term No. & 57 & 173 & 78 & 365 \\
\hline C. jejuni Y2H PPI & $47.37 \%$ & $17.01 \%$ & $16.27 \%$ & $17.91 \%$ \\
\hline C. jejuni random PPI & $35.61 \%$ & $11.04 \%$ & $12.91 \%$ & $13.97 \%$ \\
\hline Info GO ratio of C. jejuni (Y2H PPI / random) & 1.33 & 1.54 & 1.26 & 1.28 \\
\hline Info GO term No. & 3 & 26 & 22 & 51 \\
\hline Synechocystis Y2H PPI & $80.77 \%$ & $26.16 \%$ & $25.30 \%$ & $29.47 \%$ \\
\hline Synechocystis random PPI & $44.94 \%$ & $6.89 \%$ & $8.59 \%$ & $9.73 \%$ \\
\hline Info GO ratio of Synechocystis (Y2H PPI / random) & 1.80 & 3.79 & 2.95 & 3.03 \\
\hline Info GO term No. & 3 & 30 & 30 & 63 \\
\hline M. tuberculosis B2H PPI & $38.46 \%$ & $14.36 \%$ & $9.57 \%$ & $13.83 \%$ \\
\hline M. tuberculosis random PPI & $38.30 \%$ & $11.35 \%$ & $8.35 \%$ & $11.51 \%$ \\
\hline Info $\mathrm{GO}$ ratio of $\mathrm{M}$. tuberculosis (B2H PPI / random) & 1.00 & 1.26 & 1.15 & 1.20 \\
\hline Info GO term No. & 3 & 32 & 32 & 67 \\
\hline
\end{tabular}

The table is a summary of the percentage of PPIs having coherent informative GO terms, number of informative GO terms and "Info GO ratio" in each of the representative two-hybrid PPI datasets in four organisms.

Info GO ratio = percentage of PPIs in two-hybrid PPI dataset having coherent informative GO terms / percentage of PPIs in random PPI dataset having coherent informative $\mathrm{GO}$ terms. 


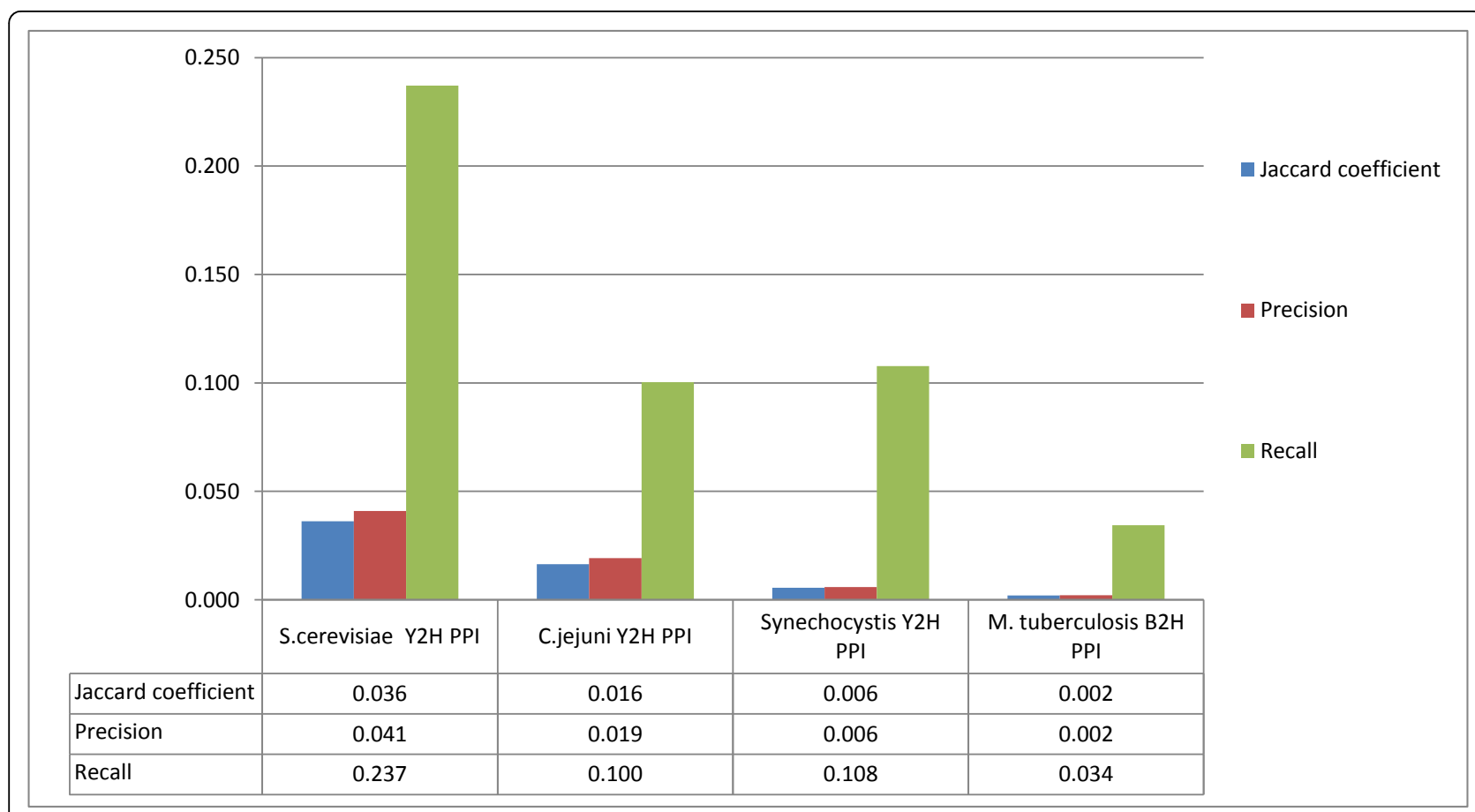

Figure 7 Similarity of representative two-hybrid PPI datasets to corresponding STRING predicted functional associations datasets. The histogram is a summary of calculated Jaccard coefficient, precision and recall between a representative two-hybrid PPI dataset (as benchmark) and its corresponding STRING predicted functional associations dataset (as testing dataset).

relationships are very few. We calculate the Jaccard coefficient, precision, and recall of each of the M. tuberculosis H37Rv PPI datasets discussed earlier using ECrel (Figure 8) and PPrel (Figure 9) from M. tuberculosis H37Rv integrated pathway gene pair relationships as the benchmarks.

Results from above experiments show that the $M$. tuberculosis H37Rv B2H PPI dataset shows very low similarity with ECrel dataset, while most of STRING PPI datasets show good similarity. This provides another explanation for the low similarity between the H37Rv B2H and STRING PPI datasets. Namely, the former dataset contains direct physical interactions, as it is to be expected of $\mathrm{B} 2 \mathrm{H}$ assays; while the latter STRING datasets also include substantial amounts of PPIs that are indirect protein relationships.

\section{STRING PPI dataset analysis in S. cerevisiae}

The comparative analysis of the various H37Rv PPI datasets using integrated pathway gene pair relationships reveals that the H37Rv STRING PPI dataset may contain a lot of indirect protein relationships. The STRING database has proclaimed itself as a database consisting of "known and predicted protein-protein interactions" [21]. In practise, both physical interactions and functional associations, and both predicted and experimental "PPIs" are included in this database. Therefore, it is important to clearly demonstrate which kind of PPIs are contained in STRING. We return to the most comprehensively investigated model organism-S. cerevisiae to more precisely analyze the characteristics of PPIs in STRING. As a unified database, the PPIs prediction approaches in the STRING database are consistently used on all the 630 organisms included in it. Thus the phenomena discovered in M. tuberculosis H37Rv should also exist in other organisms like $S$. cerevisiae, and vice versa. Moreover, we have much more information in $S$. cerevisiae that can be used for conducting a much more precise analysis. If the situation observed earlier that the

Table 7 Four types of gene relationships in integrative pathway gene pair relationships

\begin{tabular}{ll}
\hline Unified genes relationships & Explanation \\
\hline ECrel & Enzyme-enzyme relation, indicating two enzymes catalyzing successive reaction steps \\
PPrel & Protein-protein interaction, such as binding and modification, or proteins belong to same complex \\
GErel & Gene expression interaction, indicating relation of transcription factor and target gene product \\
PCrel & Protein-compound interaction \\
\hline
\end{tabular}

The detail explanations of the types of relationships in integrated pathway gene pair relationships are given below. 
Table 8 Summary of number of pathways before and after integration

\begin{tabular}{lll}
\hline Pathways source name & No. of pathways before integration & No. of unique pathways after integration \\
\hline WikiPathways & 5 pathways & 1 pathways \\
BioCyc (MTBRVCyc) & 191 pathways & 129 pathways \\
KEGG & 80 pathways & 66 pathways \\
Integrated Pathways & & 34 pathways \\
\hline
\end{tabular}

The table below shows the number of pathways from major pathway databases before and after integration.

M. tuberculosis H37Rv STRING PPI dataset contains a lot of indirect PPIs is also observe in S. cerevisiae, then it will be a sound confirmation of our earlier conclusion.

We similarly obtain the integrated pathway gene pair relationships (mainly ECrel and PPrel) for S. cerevisiae and also separate datasets prepared only from KEGG [12] for more precise reference. We further collect all protein pairs (named the "S. cerevisiae Complex PPI dataset") that appear in the same protein complexes using the protein complexes dataset from Wodak Lab [11]. It is obvious that the "S. cerevisiae Complex PPI dataset" may contain a lot of indirect PPIs, like relationships between two non-directly-binding proteins in protein complexes. A representative $S$. cerevisiae two-hybrid PPI dataset [10] is also included in this comparative analysis.

To avoid a biased comparison, as the full STRING PPI dataset may include many PPIs from the datasets above, we use the $S$. cerevisiae predicted functional associations dataset from STRING database as the testing dataset in this analysis. The overlapping number of PPIs, Jaccard coefficient, precision and recall are calculated, and the results are given in Figure 10. From the results, the $S$. cerevisiae two-hybrid PPI dataset has the lowest similarity to the S. cerevisiae STRING predicted functional associations dataset, whereas the complex PPI dataset and ECrel datasets (both from KEGG and from integrated pathway gene pair relationships) reveal good similarity to the S. cerevisiae STRING predicted functional associations dataset. This result is in accordance with the results on $M$. tuberculosis H37Rv, clearly demonstrating that the STRING database PPIs include a substantial amount of PPIs that are indirect protein relationships, including protein pairs in the same protein complexes and protein pairs catalyzing successive enzymatic reaction steps.

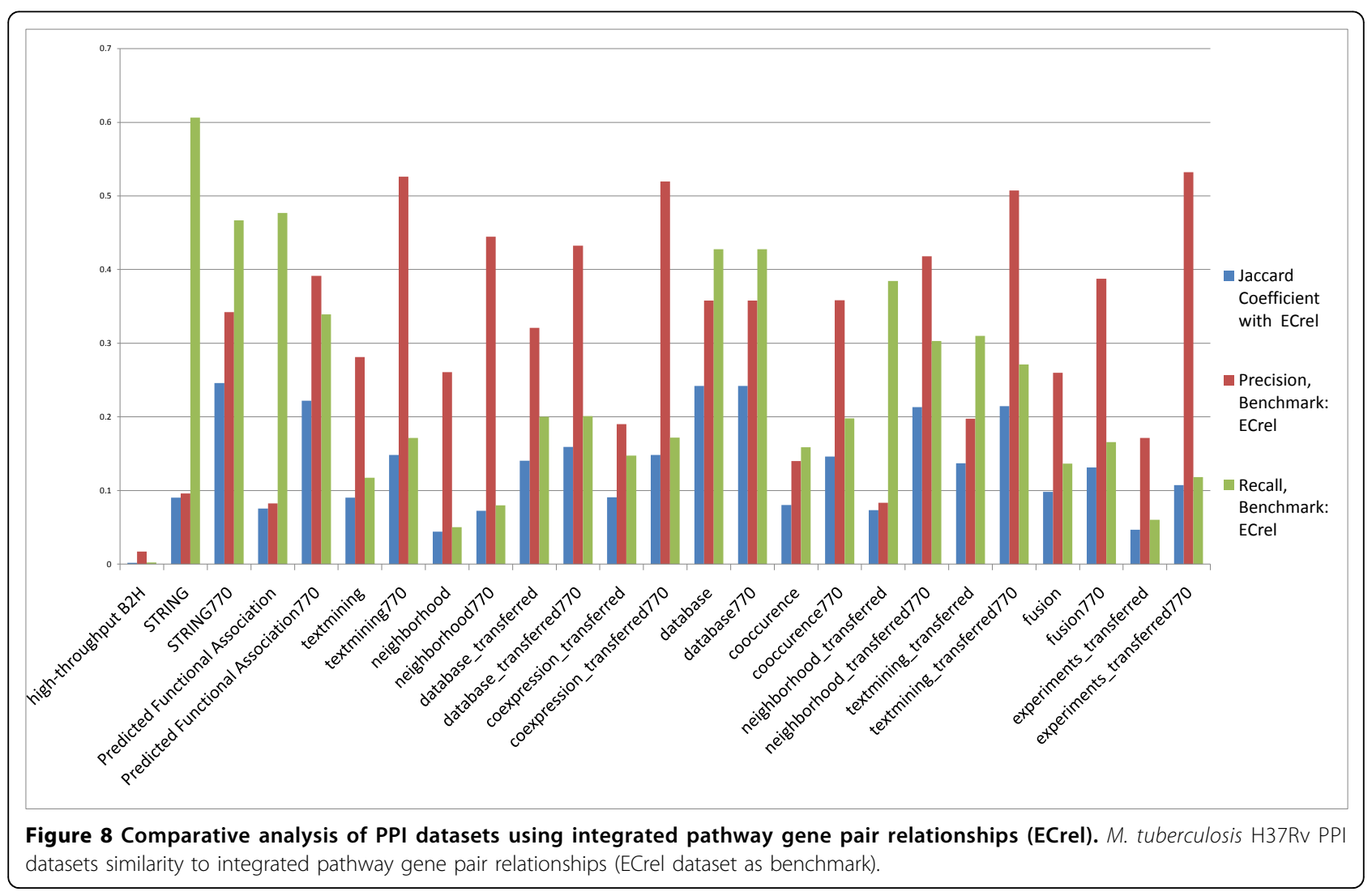




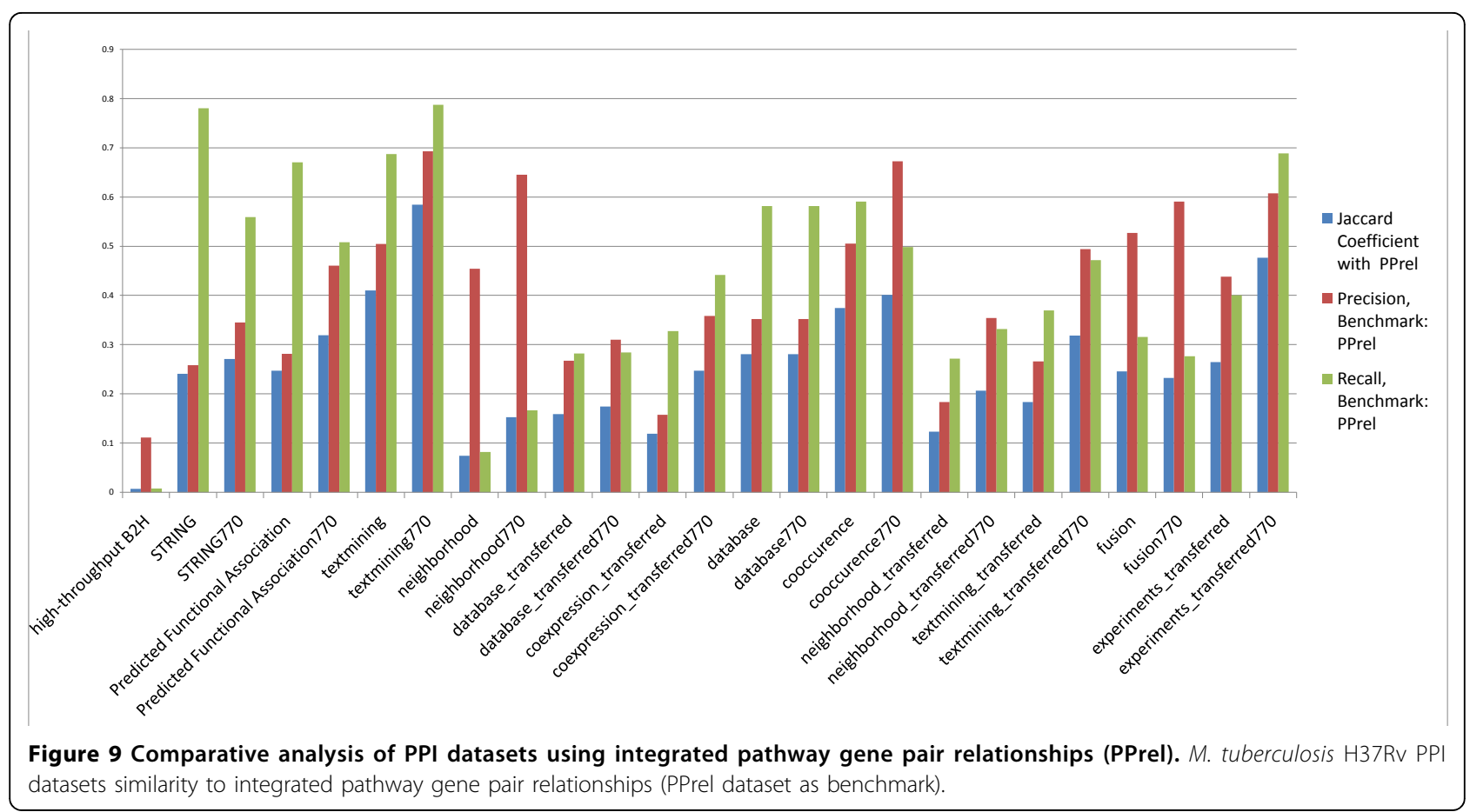

\section{Discussion}

\section{Reliable $M$. tuberculosis H37Rv B2H PPI datasets}

We have shown that the M. tuberculosis $\mathrm{H} 37 \mathrm{Rv} \mathrm{B} 2 \mathrm{H}$ PPI dataset has low quality. In the process, we find four subsets of the B2H PPI dataset that may be more reliable than the rest of this dataset. The first subset consists of PPIs where both interaction partners have coherent informative GO terms-viz., B2H PPIs sharing functional homogeneity or localization coherence. This subset contains 115 PPIs and is named "B2HsameGO dataset". The second contains overlapping PPIs between the H37Rv B2H and STRING PPI datasets, which can

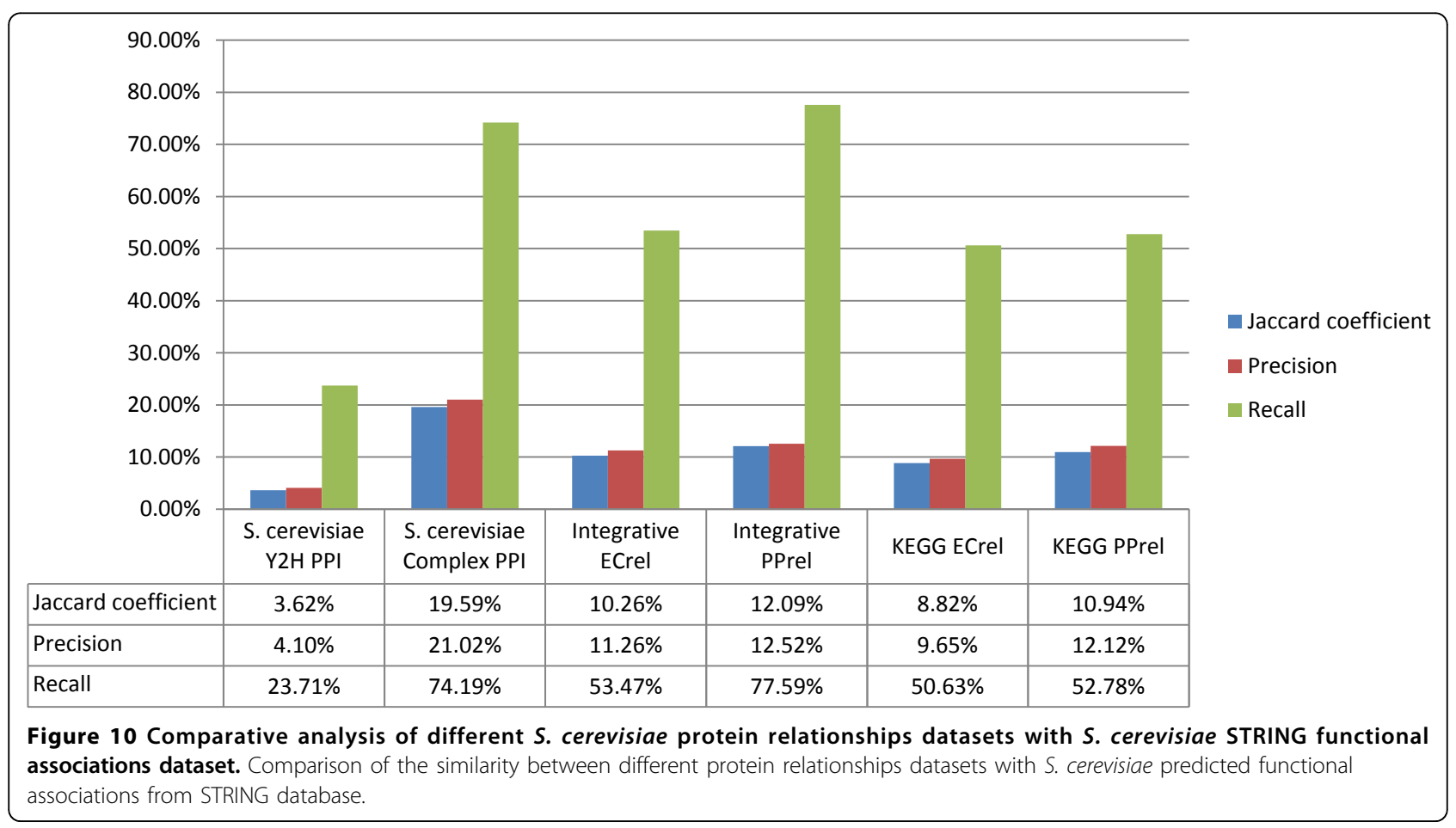


be considered as B2H PPIs supported by STRING prediction approaches. This subset consists of 276 PPIs and is named "B2HSTRING dataset". The third subset contains those H37Rv B2H PPIs that have been verified by different experiments [7]. This subset has 147 PPIs and is named "B2HdiffExp dataset". The fourth subset contains PPIs where both interaction partners have significant correlated gene expression profile (Pearson's correlation coefficient $>0.4$ ). This subset has 223 PPIs and is named "B2Hco-express dataset". The overlap between any pair of these four subsets is small because the PPIs in these four subsets involve very different proteins; see Table 9. When restricted to PPIs with both interaction partners contained in two subsets being compared, the agreements are considerably high, see also Table 9. See Additional file 1 for these reliable $M$. tuberculosis $\mathrm{H} 37 \mathrm{Rv}$ B2H PPI datasets.

There are several inherent limitations of PPI data generated by two-hybrid approaches (both $\mathrm{B} 2 \mathrm{H}$ and $\mathrm{Y} 2 \mathrm{H}$ ), including PPIs that are detected between over-expressed proteins, between fusion proteins, and in a different host (Yeast or E. coli). Given the data available in this study, we are not able to clearly identify which erroneous B2H PPIs are caused by which inherent limitations of the two-hybrid system. We leave this interesting and difficult challenge to a future project.

\section{Differences between functional associations and physical interactions}

Physical interactions correspond to direct protein relationships like two proteins binding to each other. Functional associations can be both direct and indirect protein relationships; for example, two enzymes catalyzing successive reaction steps can be regarded as functional associations. This partially explains the differences between the H37Rv B2H and STRING PPI datasets, as we have demonstrated earlier.

Based on the approach used in generating the PPIs, each of the two major categories can be further divided into two parts, "experimental physical interactions" (e. g., PPIs from Y2H or co-purification); "predicted physical interactions" (e.g., interologs predicted from co-purification PPIs); "experimental functional associations" (e. g., PPIs from synthetic lethality or dosage growth defect); "predicted functional associations" (e.g., PPIs from neighbourhood or co-occurrence). Differences among PPI datasets from the four categories are inevitable, and they all have some portion of real PPIs and some noise. However, a high noise level often overwhelms the agreement between the datasets from these four categories. Real PPIs are both functional associations and physical interactions (intersect dataset), because if two proteins truly interact with each other in normal environments, the two proteins must have functional relationships. The four subsets of reliable H37Rv B2H PPIs identified by us only contain a small number of PPIs and are not enough to illuminate the whole direct physical interactome in M. tuberculosis H37Rv, See Additional file 1 for the four subsets of reliable H37Rv B2H PPIs. Although we have identified a reliable subset of STRING PPI dataset (with score $\geq 770$ ) it may contain a substantial amount of PPIs that are indirect functional associations. Some approaches [22-24] used for protein complex prediction from PPI data can potentially be used to identify physical interactions from STRING functional associations. We leave this interesting problem to a future project.

\section{Comments on the STRING database}

The H37Rv STRING PPI dataset (with score $\geq 770$ ) has its merits, especially with respect to functional associations as described earlier. However, some obvious limitations of STRING still need to be overcome in order to maximize its benefits to the community.

Firstly, STRING version 8.3 does not make the source of individual PPIs completely transparent to the public. Neither the web interface nor the downloadable "protein.link.detail.v8.3.txt" file provides a clear indication of a PPI's exact source, especially for users who lack a license for accessing the "protein.link.full.v8.3.txt" file.

Table 9 Comparative analysis of four reliable H37Rv B2H PPI subsets

\begin{tabular}{|c|c|c|c|c|c|c|c|c|c|}
\hline $\begin{array}{c}\text { Benchmark } \\
\text { (b) }\end{array}$ & $\begin{array}{c}\text { Dataset (b) PPIs } \\
\text { No. }\end{array}$ & Testing (t) & $\begin{array}{c}\text { Dataset (t) PPIs } \\
\text { No. }\end{array}$ & $\begin{array}{l}\text { Overlap PPI } \\
\text { No. }\end{array}$ & $\begin{array}{c}\text { Jaccard } \\
\text { coefficient }\end{array}$ & Precision & Recall & $\begin{array}{c}\text { Benchmark PPI } \\
\text { No. }\end{array}$ & $\begin{array}{c}\text { Testing PPI } \\
\text { No. }\end{array}$ \\
\hline $\begin{array}{l}\text { B2Hco- } \\
\text { express }\end{array}$ & 223 & B2HsameGO & 115 & 17 & 0.85 & 0.89 & 0.944 & 18 & 19 \\
\hline $\begin{array}{l}\mathrm{B} 2 \mathrm{HcO}- \\
\text { express }\end{array}$ & 223 & B2HSTRING & 276 & 27 & 0.44 & 0.77 & 0.5 & 54 & 35 \\
\hline $\begin{array}{l}\mathrm{B} 2 \mathrm{HcO}- \\
\text { express }\end{array}$ & 223 & B2HdiffExp & 147 & 4 & 0.16 & 0.27 & 0.29 & 14 & 15 \\
\hline B2HsameGO & 115 & B2HdiffExp & 147 & 0 & 0 & Null & 0 & 1 & 0 \\
\hline B2HsameGO & 115 & B2HSTRING & 276 & 11 & 0.48 & 0.73 & 0.58 & 19 & 15 \\
\hline B2HSTRING & 276 & B2HdiffExp & 147 & 6 & 0.16 & 0.19 & 0.55 & 11 & 31 \\
\hline
\end{tabular}

The table is a summary of brief comparative analysis of four reliable H37Rv B2H PPI subsets. 
For example, they can only know that a PPI comes from "co-expression" but it can actually either come from "transferred co-expression" in other organisms through homology transfer or from "co-expression" of this organism. When looking at the "protein.link.detail.v8.3. txt" file, the H37Rv STRING PPI dataset contains 4,133 PPIs from experiments, whereas only 4 of which actually come from experiments in $\mathrm{H} 37 \mathrm{Rv}$ and the rest are from "transferred experiments". When users have the license to access all the information in this database, they still do not have a clear sense of what type of PPIs are included in STRING. It claimed to consist of "known and predicted protein-protein interactions" on its website [21], though in its publications it emphasizes on "functional associations" [3]. Actually, both "physical interactions" and "functional associations", and both "predicted" and "experimental" PPIs, are included in this database. It is helpful to collect and combine all the PPIs in the STRING database; but users should be informed of each PPI's source (predicting or reporting approaches) and characteristics (physical or functional).

Second, each version of the STRING database release time is quite long, making the STRING PPI datasets incomplete and often obsolete for many organisms. Although some PPI datasets have been published months ago, like the $S$. aureus MRSA252 pull-down PPI dataset [19] and the M. tuberculosis H37Rv B2H PPI dataset [7], neither of them has been included in the STRING database current version 8.3. The difficulty of keeping the PPI data of every organism updated in this large database (containing some 630 organisms) in a timely efficient manner is understandable. We suggest the STRING database team to divide the organisms included in the database into several tiers-for tier-1 organisms, frequent updates should be maintained; for tier-2 organisms, maybe less frequent updates are needed; and so on. This differential update process within the same large database should overcome this limitation and maximize the benefits of the STRING database.

\section{Compare the quality of high-throughput PPI datasets indirectly}

Ideally, we should have used confirmed PPI data as the benchmark in evaluating the quality of the H37Rv B2H and STRING datasets. Unfortunately, such confirmed PPI data is not available in a sufficiently large scale for M. tuberculosis H37Rv. Hence, we have resorted to the rather indirect evidence presented in this paper.

Due to inherent limitations of two-hybrid approaches, both $\mathrm{B} 2 \mathrm{H}$ - and $\mathrm{Y} 2 \mathrm{H}$-generated PPI datasets usually have low quality. It is more appropriate to compare the quality of the M. tuberculosis H37Rv B2H PPI dataset with that of other B2H PPI datasets; but such large-scale reference B2H PPI datasets are currently not available in public databases. However, by comparing the quality of the B2H PPI dataset with the quality of other representative two-hybrid PPI datasets, we can still conclude that, besides inherent limitations of two-hybrid approaches, the H37Rv B2H PPI dataset has lower quality than other two-hybrid PPI datasets. This suggests that the noise introduced by individual experiments in this H37Rv B2H PPI dataset may be higher than other datasets compared.

Hopefully, in the near future, more high quality $\mathrm{B} 2 \mathrm{H}$ PPI data will be reported and more M. tuberculosis H37Rv gold standard PPI data will be confirmed. We will then be in more suitable position to address this concern in a more direct and effective way.

\section{Conclusions}

In this work, we have observed the strikingly low agreement between $M$. tuberculosis $\mathrm{H} 37 \mathrm{Rv} \mathrm{B} 2 \mathrm{H}$ and STRING PPI datasets. We have demonstrated the two main causes of this low level of agreement. The first reason is the low quality of the B2H PPI dataset, which seems to contain a significant amount of false positives as well as false negatives. The same is true of the H37Rv STRING PPI dataset as a whole, though a subset comprising PPIs with score $\geq 700$ seems more reliable. The second reason is that the STRING PPI dataset contains a substantial amount of predicted PPIs that are not direct interactions, which seem more likely to correspond to protein pairs that are in the same protein complexes or protein pairs that are catalyzing adjacent reaction steps in enzymatic pathways.

Because of the low quality of the H37Rv B2H PPI dataset, it should not be used as a gold standard to evaluate the quality of other M. tuberculosis PPI datasets, predicted or otherwise. Researchers who need to use this dataset should do so with great caution. Yet, as the only available large-scale physical interaction dataset of M. tuberculosis $\mathrm{H} 37 \mathrm{Rv}$ at the moment, even though it suffers from high noise and low quality, the direct protein physical interaction information in this dataset should not be ignored. We have identified four subsets of this B2H PPI dataset that are more reliable, which can be combined into a single dataset, which can serve as a suitable reference $\mathrm{H} 37 \mathrm{Rv}$ physical interaction dataset for many applications.

STRING score is useful for indicating which STRING PPIs have higher quality. We suggest a STRING score threshold set around 770. Nevertheless, the H37Rv STRING PPI dataset (with score $\geq 770$ ) may contain a lot of indirect protein relationships attributable to protein pairs in the same protein complexes or protein pairs forming successive reaction steps in the same biological pathways. Therefore, this dataset can be a good 
source as a functional associations reference, but it may not be the ideal choice for the purpose of studying physical interactions in M. tuberculosis H37Rv.

\section{Methods}

\section{Preparing STRING PPI datasets for analyses}

STRING database uses a combination of prediction approaches and an integration of other information (neighborhood, transferred neighborhood, gene fusion, text mining, databases, homology transfer, co-occurrence, experiments and so on). The details of PPIs generated by each of the approaches are listed in Table 1.

STRING PPIs come from a mix of experimental data; PPIs copied from public databases (e.g. KEGG and BioGRID) and predicted PPIs. So we derive from STRING a subset of predicted PPIs and name this unbiased STRING subset "predicted functional associations dataset". This dataset is derived only from the following prediction approaches: neighborhood, transferred neighborhood, gene fusion, co-occurrence, transferred co-expression, text mining, and transferred text mining.

\section{The agreement between a benchmark PPI dataset and a testing PPI dataset}

We use Jaccard coefficient, recall, and precision to measure the agreement between a benchmark PPI dataset and a testing PPI dataset. Jaccard coefficient is defined as the size of the intersection of the two datasets divided by the size of the union of the two datasets. Recall is the proportion of benchmark PPIs that are in the testing dataset. Precision is the proportion of testing PPIs that are in the benchmark dataset. Thus,

Jaccard coefficient $=\mathrm{TP} /(\mathrm{TP}+\mathrm{FP}+\mathrm{FN})$;

Precision $=\mathrm{TP} /(\mathrm{TP}+\mathrm{FP})$;

Recall $=\mathrm{TP} /(\mathrm{TP}+\mathrm{FN})$.

Here, TP (true positives) represents the number of PPIs in the testing dataset that overlap with the benchmark dataset; FN (false negatives) represents the number of PPIs in the benchmark dataset that are not in the testing dataset; TN (true negatives) represents the number of all possible PPIs that appear in neither the testing dataset nor the benchmark dataset; and FP (false positives) represents the number of PPIs in testing dataset but are not in the benchmark dataset. The Jaccard coefficient, recall, and precision of the benchmark and testing datasets considered in this work are given in Figures $1,7,8,9$, and 10 .

\section{STRING score distribution of "overlap PPI number ratio"}

In order to find which STRING score region has a higher percentage of overlapping PPIs with the B2H PPI dataset, STRING score distribution of "overlap PPI number ratio" between the STRING predicted functional associations dataset and the M. tuberculosis
$\mathrm{H} 37 \mathrm{Rv}$ B2H PPI dataset were calculated and plotted in Figure 2. At each score interval of 10, the "overlap PPI number ratio" is defined as the number of overlapping PPIs divided by the total number of PPIs in that interval. For example, if there are 300 PPIs from the STRING predicted functional associations dataset are in score range 150 160, and among these 300 PPIs there are 30 PPIs overlapping with the B2H PPI dataset, then in this score range 150 160 the "overlap PPI number ratio" is $30 / 300=0.1$. We calculate all the "overlap PPI number ratio" in each interval, STRING score ranging from 150 to 1000 , and the distribution of the ratios are plotted in Figure 2.

\section{PPI datasets analysis by PPI functional intensity matrix}

COG function annotations are used in the analysis. We calculate the percentage of PPIs in each cell in the matrix. In the matrix, each cell is a combination of the COG functional category between a pair of proteins. The percentage ("intensity") of PPIs in each cell is the number of PPIs in that cell divided by the total number of PPIs in the dataset. The number of PPIs in a certain cell means the number of PPIs fit the functional categories combination; that is, one partner has been annotated with the COG category on the $\mathrm{x}$ axis, the other partner has been annotated with the COG category on the $y$ axis. If " $\mathrm{m}$ " PPIs have "J-K" annotations in a dataset that has "n" PPIs-and "J-K" annotations means one partner form J COG functional category, the other partner from "K" COG functional category-then the PPI functional intensity in the "J-K" cell is " $\mathrm{m} / \mathrm{n}$ ". This is slightly different from the conventional approach of calculating the PPI functional intensity matrix; but it is good enough to convey broad information on the functional distribution of the PPI datasets tested. The PPI functional intensity matrices generated in this work are displayed in Figure 3.

\section{GO term annotation, informative GO term identification and PPI datasets assessments}

M. tuberculosis H37Rv, C. jejuni NCTC11168 and Synechocystis sp. PCC6803 proteins are annotated with GO terms using InterProScan [25]. GO terms are organized into three separate hierarchical ontologies-viz., cellular component terms (CC), molecular function terms (MF), and biological process terms (BP). A protein that is annotated by a particular GO term is considered to be annotated by all ancestor terms (in the corresponding hierarchical ontology) of that GO term-that is, the socalled "through-path" rule is applied. As top-level GO terms tend to be annotated to many proteins and leaflevel GO terms to very few proteins, in order to avoid bias in our analysis, we keep only "informative" GO terms for analysis. An informative GO term is defined 
as a GO term that has at least 30 proteins assigned to it or its descendants and none of its child terms have 30 or more proteins assigned to it. This way, exactly one GO term is considered in any through path. Moreover, each GO term considered is at the finest resolution possible while being annotated to a sufficiently large number of proteins $(\geq 30)$ for a valid analysis.

A pair of proteins come into contact with each other and interacts to perform a function. If the GO term annotations of the proteins in an organism are complete, we can expect such a pair of interacting proteins to have at least one informative GO term annotation in common. Therefore, a predicted or reported PPI is more likely to be a false positive when the two proteins in the PPI do not have an informative GO term annotated to them in common. We can therefore gauge the quality of a PPI dataset by calculating the percentage of PPIs in the dataset (where both proteins in the PPI have informative GO term annotations) that has "coherent" informative GO term annotations. A PPI is said to have coherent informative GO term annotation if the two proteins in the PPI have an informative GO term annotation in common.

However, the percentage of PPIs in a dataset that have coherent informative GO terms can be affected by the number of informative GO terms and by biases in the distribution of proteins these informative GO terms are annotated to. For example, if only one informative GO term was available in the organism, then $100 \%$ of the annotated PPIs would be coherent. Thus, to better assess the quality of a PPI dataset by coherence of informative GO term annotations, we need to compare the percentage of coherently annotated PPIs in the dataset to appropriately generate random PPI datasets. In particular, a high ratio (named "Info GO ratio") of the percentage of coherently annotated PPIs in the PPI dataset compared to that of the random PPI dataset suggests that PPI dataset is likely to be of high quality. We generate random PPI network using the Random Network Plug-in [26] in Cytoscape [27]. The percentage of PPIs that have coherent informative GO term annotations in the PPI datasets considered in this work is given in Figures 4 and 6 , as well as Tables 2 and 8.

\section{Gene expression profile correlation}

A pair of proteins needs to be expressed in the same location and at the same time in order to interact. Thus the highly correlated gene expression profiles of two genes suggest the likely interaction of their two gene products. Genome-wide gene expression profiles under metabolic inhibitory and control conditions [1] are used here to gauge the correlation of gene expression profiles. Given any protein pair $(x, y)$, the correlation of the expression profiles $\left(G_{x}, G_{y}\right)$ of the two underlying genes are calculated by Pearson correlation coefficient as follows[28],

$$
S_{G E}\left(G_{x}, G_{y}\right)=\frac{\left|\sum_{i=1}^{n}\left(x_{i}-\bar{x}\right)\left(y_{i}-\bar{y}\right)\right|}{\sqrt{\sum_{i=1}^{n}\left(x_{i}-\bar{x}\right)^{2}} \sqrt{\sum_{i=1}^{n}\left(y_{i}-\bar{y}\right)^{2}}} .
$$

Here, $\mathrm{n}$ is the number of conditions for the expression profiles; $x_{i}$ is the $i^{\text {th }}$ expression value of the gene corresponding to protein $\mathrm{x}$ in the gene expression profiles $\mathrm{G}_{\mathrm{x}}$; and $x$ is the average value of $\mathrm{G}_{\mathrm{x}}$.

The distributions of Pearson correlation values for PPIs in the datasets considered here are plotted in Figure 5 .

\section{M. tuberculosis $\mathrm{H} 37 \mathrm{Rv}$ interolog prediction}

An interolog is a conserved interaction between a pair of proteins which have interacting homologs in another organism. We use two different methods to identify homologs between M. tuberculosis H37Rv and the other 629 organisms in STRING. In the first method, we use Inparanoid [29] with default parameter setting. In the second method, we use BLASTP [30] with HVAL $>20$ and E-VALUE $<1 * \mathrm{e}-6$. In the third method, we use BLASTP with PIDE $>30$, length of the alignment $>20 \%$ of query sequence length, and E-VALUE $<1$ "e- 10 .

Here, the HVAL [31] is the percentage identities threshold based on the HSSP curve. It is defined as:

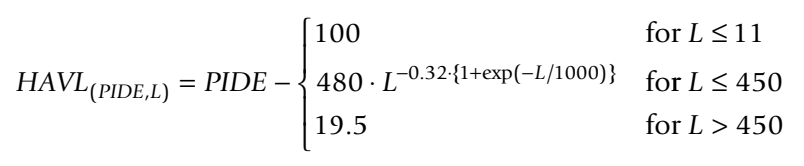

where PIDE is the percentage identities of the BLASTP hit and $\mathrm{L}$ is the length of the alignment.

\section{Analysis of characteristics of PPI datasets using integrated pathway gene pair relationships}

In order to better understand what the predicted PPIs in STRING really correspond to, we use the pair-wise relationships of proteins/genes from the XML files of each of the pathways in KEGG [12], WikiPathways [13,14] and BioCyc [15]. The XML files in these three major pathway databases follow different formats. KEGG pathways information is stored in KGML format. WikiPathways $[13,14]$ pathway information is recorded in GPML format. BioCyc (specifically, MTBRVcyc) pathway information is stored in BioPAX format [32]. Mining and retrieving pair-wise protein/gene relationships from KGML and GPML are mainly by using our in-house program. For BioPAX [32], we convert BioPAX files into simple SIF files use paxtools [32]; then we make some node mapping to retrieve the protein/gene pair 
relationships. These gene pair relationships are classified in accordance to KEGG [12] into four categories: ECrel, PPrel, GErel, PCrel; see Table 7. In particular, ECrel comprises protein/gene pairs that catalyze/form adjacent reaction steps in enzymatic pathways, and PPrel comprises protein-protein interactions such as binding and modifications.

Software packages, program tools, materials and datasets The software packages and program tools used in this study are:

(a) Protein Sequence Alignment software package: BLASTP [30,33].

(b) Orthologues Identification software package:

Inparanoid stand-alone package, Version 4.1 [29].

(c) BioPAX pathway file processing software package:

Paxtools [32].

(d) Random PPI network generation:

Random Network Plug-in [26] for Cytoscape [27].

(e) Protein Signature annotation program:

InterProScan [25].

The datasets used in this study are:

(a) M. tuberculosis H37Rv Gene Expression Datasets [1].

(b) Pathways Datasets:

KEGG [12].

WikiPathways [13].

BioCyc [15].

(c) PPI datasets:

M. tuberculosis $\mathrm{H} 37 \mathrm{Rv}$ high-throughput B2H PPI dataset [7].

M. tuberculosis H37Rv in STRING [3].

S. cerevisiae protein complexes dataset obtained from Wodak Lab [11].

S. cerevisiae two-hybrid PPI dataset [10].

C. jejuni two-hybrid PPI dataset [8].

Synechocystis two-hybrid PPI dataset [9].

(d) M. tuberculosis $\mathrm{H} 37 \mathrm{Rv}$ proteins function annotations:

COG functional categories annotation [34].

(e) S. cerevisiae GO annotations downloaded from Gene Ontology website [35] dated 6/12/2010.

\section{Additional material}

Additional file 1: Reliable M. tuberculosis H37Rv B2H PPI datasets. Description: the reliable M. tuberculosis $\mathrm{H} 37 \mathrm{Rv} \mathrm{B} 2 \mathrm{H}$ PPI datasets, list in four text files, tab delimited.

\section{Acknowledgements}

We thank Chern Han Yong and Michał Woźniak for critical discussions and suggestions. We thank also Igor Rodchenkov of the Pathway Commons Team and BioPAX Core Group for help and instructions on using paxtool packages effectively. This project was supported in part by an NGS scholarship and a Singapore Ministry of Education Tier-2 grant MOE2009-T22-004.

This article has been published as part of BMC Genomics Volume 12 Supplement 3, 2011: Tenth International Conference on Bioinformatics - First ISCB Asia Joint Conference 2011 (InCoB/ISCB-Asia 2011): Computational Biology. The full contents of the supplement are available online at http:// www.biomedcentral.com/1471-2164/12?issue=S3.

\section{Author details}

${ }^{1}$ NUS Graduate School for Integrative Sciences \& Engineering, National University of Singapore, 28 Medical Drive, Singapore $117456 .{ }^{2}$ School of Computing, National University of Singapore, 13 Computing Drive, Singapore 117417 .

\section{Authors' contributions}

This work was jointly conceived, planned, analyzed, and written up by LW and $\mathrm{HZ}$. The analytical experiments were performed by $\mathrm{HZ}$.

\section{Competing interests}

The authors declare that they have no competing interests.

\section{Published: 30 November 2011}

\section{References}

1. Boshoff HIM, Myers TG, Copp BR, McNeil MR, Wilson MA, Barry CE: The transcriptional responses of Mycobacterium tuberculosis to inhibitors of metabolism. Journal of Biological Chemistry 2004, 279:40174.

2. Cole ST, Brosch R, Parkhill J, Garnier T, Churcher C, Harris D, Gordon S, Eiglmeier K, Gas S, Barry CE: Deciphering the biology of Mycobacterium tuberculosis from the complete genome sequence. Nature 1998, 393:537-544.

3. Jensen $L$, Kuhn M, Stark M, Chaffron S, Creevey C, Muller J, Doerks T, Julien P, Roth A, Simonovic M: STRING 8-a global view on proteins and their functional interactions in 630 organisms. Nucleic acids research 2009, 37:D412.

4. Raman K, Yeturu K, Chandra N: targetTB: a target identification pipeline for Mycobacterium tuberculosis through an interactome, reactome and genome-scale structural analysis. BMC systems biology 2008, 2:109.

5. Raman K, Chandra N: Mycobacterium tuberculosis interactome analysis unravels potential pathways to drug resistance. BMC microbiology 2008, 8:234.

6. Li AH, Waddell SJ, Hinds J, Malloff CA, Bains M, Hancock RE, Lam WL, Butcher PD, Stokes RW: Contrasting transcriptional responses of a virulent and an attenuated strain of Mycobacterium tuberculosis infecting macrophages. PloS one 2010, 5:e11066.

7. Wang Y, Cui T, Zhang C, Yang M, Huang Y, Li W, Zhang L, Gao C, He Y, $\mathrm{Li} Y$, et al: Global protein-protein interaction network in the human pathogen Mycobacterium tuberculosis H37Rv. J Proteome Res 2010, 9:6665-6677.

8. Parrish JR, Yu J, Liu G, Hines JA, Chan JE, Mangiola BA, Zhang H, Pacifico S, Fotouhi F, DiRita VJ: A proteome-wide protein interaction map for Campylobacter jejuni. Genome Biology 2007, 8:R130.

9. Sato S, Shimoda Y, Muraki A, Kohara M, Nakamura Y, Tabata S: A large-scale protein-protein interaction analysis in Synechocystis sp. PCC6803. DNA research 2007, 14:207.

10. Yu H, Braun P, Y Id r m MA, Lemmens I, Venkatesan K, Sahalie J, HirozaneKishikawa T, Gebreab F, Li N, Simonis N: High-quality binary protein interaction map of the yeast interactome network. Science 2008, 322:104.

11. Pu S, Wong J, Turner B, Cho E, Wodak SJ: Up-to-date catalogues of yeast protein complexes. Nucleic acids research 2009, 37:825.

12. Kanehisa M, Goto S: KEGG: Kyoto encyclopedia of genes and genomes. Nucleic acids research 2000, 28:27.

13. Pico AR, Kelder T, Van lersel MP, Hanspers K, Conklin BR, Evelo C: WikiPathways: pathway editing for the people. PLoS biology 2008, 6:e184.

14. Kelder T, Pico AR, Hanspers K, Van lersel MP, Evelo C, Conklin BR: Mining biological pathways using WikiPathways web services. PloS one 2009, 4: e6447.

15. Karp PD, Ouzounis CA, Moore-Kochlacs C, Goldovsky L, Kaipa P, Ahrén D, Tsoka S, Darzentas N, Kunin V, López-Bigas N: Expansion of the BioCyc collection of pathway/genome databases to 160 genomes. Nucleic acids research 2005, 33:6083. 
16. Chua HN, Sung WK, Wong L: Exploiting indirect neighbours and topological weight to predict protein function from protein-protein interactions. Bioinformatics 2006, 22:1623.

17. Grigoriev A: A relationship between gene expression and protein interactions on the proteome scale: analysis of the bacteriophage $\mathrm{T7}$ and the yeast Saccharomyces cerevisiae. Nucleic acids research 2001 29:3513.

18. Gene Expression Omnibus. [http://www.ncbi.nlm.nih.gov/geo/]

19. Cherkasov A, Hsing M, Zoraghi R, Foster LJ, See RH, Stoynov N, Jiang J, Kaur $S$, Lian $T$, Jackson $L$, et al: Mapping the protein interaction network in methicillin-resistant Staphylococcus aureus. J Proteome Res 2011, 10:1139-1150.

20. Aranda B, Achuthan P, Alam-Faruque $Y$, Armean I, Bridge A, Derow C Feuermann M, Ghanbarian A, Kerrien S, Khadake J: The IntAct molecular interaction database in 2010. Nucleic acids research 2010, 38:D525.

21. STRING. [http://string-db.org/]

22. Qiu J, Noble WS: Predicting co-complexed protein pairs from heterogeneous data. PLoS computational biology 2008, 4:e1000054.

23. Wang H, Kakaradov B, Collins SR, Karotki L, Fiedler D, Shales M, Shokat KM Walther TC, Krogan NJ, Koller D: A complex-based reconstruction of the Saccharomyces cerevisiae interactome. Molecular \& Cellular Proteomics 2009, 8:1361.

24. Zhang L, Wong S, King O, Roth F: Predicting co-complexed protein pairs using genomic and proteomic data integration. BMC bioinformatics 2004, 5:38.

25. Zdobnov EM, Apweiler R: InterProScan-an integration platform for the signature-recognition methods in InterPro. Bioinformatics 2001, 17:847.

26. Random Network Plugin. [http://sites.google.com/site/ randomnetworkplugin/].

27. Shannon P, Markiel A, Ozier O, Baliga NS, Wang JT, Ramage D, Amin N, Schwikowski B, Ideker T: Cytoscape: a software environment for integrated models of biomolecular interaction networks. Genome research 2003, 13:2498.

28. Wu M, Li X, Chua H, Kwoh CK, Ng SK: Integrating diverse biological and computational sources for reliable protein-protein interactions. BMC bioinformatics 2010, 11:S8.

29. Remm M, Storm CEV, Sonnhammer ELL: Automatic clustering of orthologs and in-paralogs from pairwise species comparisons1. Journal of molecular biology 2001, 314:1041-1052.

30. Altschul SF, Madden TL, Schäffer AA, Zhang J, Zhang Z, Miller W, Lipman DJ: Gapped BLAST and PSI-BLAST: a new generation of protein database search programs. Nucleic acids research 1997, 25:3389.

31. Rost B: Twilight zone of protein sequence alignments. Protein engineering $1999,12: 85$

32. Demir E, Cary MP, Paley S, Fukuda K, Lemer C, Vastrik I, Wu G, D'Eustachio P, Schaefer C, Luciano J: The BioPAX community standard for pathway data sharing. Nature biotechnology 2010, 28:935-942.

33. NCBI/BLAST Home. [http://blast.ncbi.nlm.nih.gov/Blast.cgi? CMD $=$ Web\&PAGE_TYPE $=$ BlastHome].

34. Clusters of Orthologous Groups of proteins (COGs). [http://www.ncbi.nlm. nih.gov/COG/1.

35. Gene Ontology website. [http://www.geneontology.org/].

\section{Submit your next manuscript to BioMed Central and take full advantage of:}

- Convenient online submission

- Thorough peer review

- No space constraints or color figure charges

- Immediate publication on acceptance

- Inclusion in PubMed, CAS, Scopus and Google Scholar

- Research which is freely available for redistribution

Submit your manuscript at www.biomedcentral.com/submit
C Biomed Central 